\title{
Shape Theory of Maps
}

\author{
Zvonko C̈ERIN
}

\begin{abstract}
We shall describe a modification of homotopy theory of maps which we call shape theory of maps. This is accomplished by constructing the shape category of maps $\mathcal{H} M^{b}$. The category $\mathcal{H} M^{b}$ is built using multi-valued functions. Its objects are maps of topological spaces while it's morphisms are homotopy classes of collections of pairs of multi-valued functions which we call multi-binets. Various authors have previously given other descriptions of shape categories of maps. Our description is intrinsic in the sense that we do not use any outside objects. It is a version of the author's extension to arbitrary topological spaces of Sanjurjo's approach to shape theory via small multi-valued functions adapted to maps.
\end{abstract}

\section{INTRODUCTION}

It is quite often in topology that a concept that was introduced for spaces is later also considered for maps. An example relevant to this paper is provided by the classical homotopy category $\mathcal{H}$ on the one hand and the homotopy category of maps $\mathcal{H}^{b}$ from E. H. Spanier's book on algebraic topology [10] on the other. The objects of $\mathcal{H}$ are topological

1991 Mathematics Subject Classification: 54B25, 54F45, 54C56

Servicio publicaciones Univ. Complutense. Madrid, 1995. 
spaces and morphisms are homotopy classes of maps between them while the objects of $\mathcal{H}^{b}$ are maps of topological spaces and morphisms are homotopy classes of map pairs between them. In this way we can do homotopy theory of maps and talk about contractible maps, homotopy type of maps, and other notions from homotopy theory transferring them from spaces to maps.

The classical homotopy theory has been modified by the introduction of shape theory. The modification was invented by K. Borsuk [3] with the desire to handle more successfully spaces with bad local properties. The new improved homotopy theory that he named shape theory agrees with the old on spaces with nice local properties, for example on the absolute neighbourhood retracts. The key idea in Borsuk's approach was to replace homotopy classes of maps with homotopy classes of sequences of maps that he calls fundamental sequences.

The aim of this paper is to define shape theory for maps of topological spaces. In other words, we shall describe a shape category of maps $\mathcal{H} M^{b}$ whose objects are maps of topological spaces and whose morphisms will be homotopy classes of pairs of collections of multi-valued functions that. we call multi-binets. Therefore, our approach can be regarded as a version for maps of the author's description [4] of the shape category $S h$ which is an extension of Sanjurjo's method [9] from compact metric spaces to arbitrary topological spaces.

Oür construction extends shape theory from 'spaces to maps because the full subcategory of $\mathcal{H} M^{b}$ with maps of topological spaces into a single-element space as objects is naturally isomorphic to the shape category $\mathcal{S} h[8]$.

The following features of our description of shape category of maps deserve to be emphasized. It is intrinsic in the sense that we do not use any outside objects (like ANR's, embeddings, resolutions, and expansions). It is simple and geometric because it is modelled on the original Borsuk's description of shape theory of compact metric spaces [3]. The only difficult part is in defining the composition of morphisms.

There are already two previous attempts to define shape theory of maps in the same generality as ours. In [6], David Edwards and Patricia McAuley use both Cech and Vietoris constructions to approximate a map $f: X \rightarrow Y$ by a tower of maps between $C W$-complexes. Each map 
gives in this way an object of a suitable pro-category. The study of the shape properties of a map is thus reduced to the study of this associated pro-object. This approach is useful because one can apply homotopy invariant functors (like homology, cohomology, and homotopy groups functors) to get various pro-groups which reflect the geometry of the map.

The second attempt is similar and is due to V.H. Baladze. In [2], he exhibits an outline without any proofs of a shape theory of maps of arbitrary topological spaces following the inverse limit approach to shape theory based on the method of ANR-resolutions, i.e., resolutions of spaces consisting of absolute neighbourhood retracts. In this way a greater flexibility is achieved because we are no longer forced to use Cech and Vietoris constructions that are plagued by certain inconveniences. However, it is extremely complicated to find an $\operatorname{ANR}\left(M_{m a p s}\right)$-expansion for a map, which is a prerequisite for a study of shape properties of the map by this method. Both [6] and [2] belong to the so called outer shape theory in which shape of spaces and maps is investigated with the help of some outside objects like expansions into absolute neighbourhood retracts.

The natural questions of relationship of our shape theory of maps with those mentioned above are deferred to another paper. As an illustration of our method, we shall discuss the problem of identifying maps on which shape theory of maps and homotopy theory of maps coincide. Some interesting classes of maps which we call internally movable, internally calm, and calm provide an unexpected solution to this question. We also give some applications of our approach to identify equivalences in the shape category of maps and maps with trivial shape.

Here is a brief outline of our organization. The principal goal of the paper is to describe with all details the category $\mathcal{H} M^{b}$. Homotopy theory of maps which we shall be improving is recalled first. Since our method relies on the idea of approximating a map by a generalized sequence (net over a cofinite directed set) of multi-valued functions with smaller and smaller images of points, we present facts on normal covers [1] which we use as a mean of measuring size of sets and from which our sets of indices for these nets are build. Connection between indexing sets of our nets provide increasing functions which are normally constructed by a well-known simple Lemma 1 (see $[8$, p. 9]). 
Then we define multi-valued functions and multi-valued $\sigma$-functions (i.e. a multi-valued function of size less than a normal cover $\sigma$ of the codomain). Our multi-valued functions must satisfy only the condition that they associate a non-empty subset of the codomain to every point of the domain.

The next step is to define all of these notions for maps. This is done by considering pairs and replacing exact commutativity with an approximate form of it up to a normal cover in the codomain. Since all concepts dealing with maps usually involve pairs we take the convention that they include prefix "bi" in their names. Thus maps themselves are also called bispaces and we define notions of bimap, multi-valued bifunction, normal bicover, and others. The multi-valued $\sigma$-functions for maps (or multi-valued $\sigma$-bifunctions) lead naturally to the relation of $\sigma$-bihomotopy. The important approximate transitivity of this relation is established in Lemma 2. In the first reading one can skip it's tedious proof with many cases provided one is familiar with Dold's powerful lemma (see [5, p. 358]) on which our argument depends and which is used later over and over again.

After these preliminaries, we follow closely Borsuk's method from [3]. The role of fundamental sequences play multi-binets, i.e., multi-nets for maps. We define the relation of bihomotopy for multi-binets and take as objects of our category $\mathcal{H} M^{b}$ maps and as morphisms bihomotopy classes of multi-binets. In order to prove that this is indeed a category we must describe how one composes morphisms and one must prove that this composition is associative. This is done in Claims 1,2 , and 3 and Theorem 1. The details are quite easy once the method of proof for relations (4) and (5) is understood. It would be unreal to expect that in the complete generality which we consider here one can get the shape category of maps without any effort.

The rest of the paper presents some applications of our approach to shape theory of maps. They are included with intention to convince the reader that the new method will be useful for some kind of problems. We intend to undertake further study of the category $\mathcal{H} \boldsymbol{M}^{b}$. This paper is only the beginning and it gives basic facts about this approach to shape theory for maps. 


\section{HOMOTOPY THEORY OF MAPS}

Since shape theory is just a modification of homotopy theory, we begin by recalling some notions from homotopy theory of maps.

We follow the convention that a map is a short name for a continuous single-valued function. In order to get closer analogies in our constructions, we shall also use bispace as another name for a map. More formally, a bispace is a triple $\left(f, F^{\prime}, F\right)$ consisting of topological spaces $F^{\prime}$ and $F$ and a continuous single-valued function $f: F^{\prime} \rightarrow F$. Since we shall scrupulously follow the notation that a map is denoted by a small letter, it's domain and codomain with the same capital letter, and all domains have in addition a prime, we shall drop $F^{\prime}$ and $F$ from our notation and use $f$ to denote a bispace $\left(f, F^{\prime}, F\right)$. However, more important for our exposition will be the fact that a presence of a prefix "bi" and either a superscript or a subscript " $b$ " indicates something referring to maps. Thus, $x, X^{b}$, and bispace $X$ are all notations for the map $\left(x, X^{\prime}, X\right)$. With these convention enforced consistently we shall see that most results from [4] carry over to maps. In all statements and proofs in [4] we must only put bi and $b$ at proper places to get shape theory for maps.

Following $\left[10 ;\right.$ p. 414], we now consider the category $S^{b}$ whose objects are bispaces (i.e., maps $f: F^{\prime} \rightarrow F$ between topological spaces) and whose morphisms will be called bimaps. Let $F$ and $G$ be bispaces. By a bimap from $F$ into $G$ we mean a pair $\left(a^{\prime}, a\right)$ of maps $a^{\prime}: F^{\prime} \rightarrow G^{\prime}$ and $a: F \rightarrow G$ such that $g \circ a^{\prime}=a \circ f$. In other words we require that the following diagram commutes.

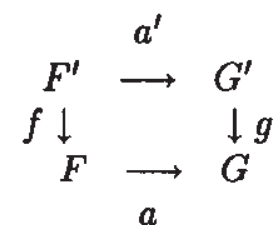

Just as with bispaces, we shall also simplify our notation for bimaps. Thus "... bimap $a: F \rightarrow G \ldots$ " means that we have a bimap $\left(a^{\prime}, a\right)$ between bispaces $F$ and $G$. The composition of bimaps $\left(a^{\prime}, a\right)$ and $\left(b^{\prime}, b\right)$ 
is a bimap $\left(b^{\prime} \circ a^{\prime}, b \circ a\right)$ and the identity bimap $1_{X}$ on a bispace $X$ is a bimap $\left(1_{X^{\prime}}, 1_{X}\right)$, where $1_{X}$ is the identity map on the space $X$.

The definition of bihomotopy involves the bicylinder $F \times I$ of a bispace $F$. Let $I$ denote the unit closed segment and let $I$ denote also the bispace $\left(1_{I}, I, I\right)$, where $1_{I}: I \rightarrow I$ is the identity map. The bicylinder is a special case of the biproduct defined for bispaces $F$ and $G$ to be the bispace ( $f \times g, F^{\prime} \times G^{\prime}, F \times G$ ).

Let $p$ and $q$ be bimaps between bispaces $X$ and $Y$. We shall say that $p$ and $q$ are bihomotopic and write $p \simeq_{b} q$ provided there is a bimap $m: X \times I \rightarrow Y$ called bihomotopy such that $m^{\prime}\left(a^{\prime}, 0\right)=p^{\prime}\left(a^{\prime}\right)$ and $m^{\prime}\left(a^{\prime}, 1\right)=q^{\prime}\left(a^{\prime}\right)$ for every $a^{\prime} \in X^{\prime}$ and $m(a, 0)=p(a)$ and $m(a, 1)=$ $q(a)$ for every $a \in X$. The relation of bihomotopy is an equivalence relation and we denote the bihomotopy class of a bimap $p$ by $[p]_{b}$. Since the bihomotopy relation $\simeq_{b}$ is also compatible with the composition of bimaps, one can define the composition of bihomotopy classes of bimaps by composing representatives, i.e., $[p]_{b} \circ[q]_{b}=[p \circ q]_{b}$. In this way one obtains the bihomotopy category $\mathcal{H}^{b}$, whose objects are topological bispaces and whose morphisms are bihomotopy classes of bimaps. There is a bihomotopy functor $H^{b}$ from $S^{b}$ to $\mathcal{H}^{b}$ which keeps the objects fixed and takes a bimap $p$ into it's bihomotopy class $[p]_{b}$.

\section{NORMAL COVERS AND MULTI-VALUED FUNCTIONS}

In this section we shall introduce notions and results on normal covers and multi-valued functions that are required for our theory.

Let $\operatorname{Cov}(Y)$ denote the collection of all normal covers of a topological space $Y[1]$. With respect to the refinement relation $\geq$ the set $\operatorname{Cov}(Y)$ is a directed set. Two normal covers $\sigma$ and $\tau$ of $Y$ are equivalent provided $\sigma \geq \tau$ and $\tau \geq \sigma$. In order to simplify our notation we denote a normal cover and it's equivalence class by the same symbol. Consequently, $\operatorname{Cov}(Y)$ also stands for the associated quotient set.

If $\sigma$ is a normal cover of a space $Y$, let $\sigma^{+}$be the collection of all normal covers of $Y$ which refine $\sigma$ while $\sigma^{*}$ denotes the set of all normal covers $\tau$ of $Y$ such that the star st $(\tau)$ of $\tau$ refines $\sigma$. Similarly, for a natural number $n, \sigma^{* n}$ denotes the set of all normal covers $\tau$ of $Y$ such that the $n$-th star $s t^{n}(\tau)$ of $\tau$ refines $\sigma$. 
Let $\operatorname{Inc}(Y)$ denote the collection of all finite subsets $c$ of $\operatorname{Cov}(Y)$ which have a unique (with respect to the refinement relation) maximal element which we denote by [c]. The notation $\operatorname{Inc}(Y)$ comes from "indices of covers". The set $\operatorname{Inc}(Y)$ will be used as indexing set for multi-nets into $Y$. We consider $\operatorname{Inc}(Y)$ ordered by the inclusion relation and regard $\operatorname{Cov}(Y)$ as a subset of single-element subsets of $C o v(Y)$. Notice that $\operatorname{Inc}(Y)$ is a cofinite directed set.

We shall repeatedly use the following lemma (see [8, p. 9]). Let us agree that an increasing function $f: P \rightarrow P$ of a partially ordered set $(P, \leq)$ into itself is a function which satisfies $x \leq f(x)$ for every $x \in P$ and $x \leq y$ in $P$ implies $f(x) \leq f(y)$. In the case when the domain and the codomain of a function $f$ are different, the first requirement is dropped.

Lemma 1. Let $\left\{f_{1}, \ldots, f_{n}\right\}$ be functions from a cofinite directed set $(M, \leq)$ into a directed set $(L, \leq)$. Then there is an increasing function $g: M \rightarrow L$ such that $g(x) \geq f_{1}(x), \ldots, f_{n}(x)$ for every $x \in M$.

Let $X$ and $Y$ be topological spaces. By a multi-valued function $F: X \rightarrow Y$ we mean a rule which associates a non-empty subset $F(x)$ of $Y$ to every point $x$ of $X$.

For our approach to shape theory the following notion of size for multi-valued functions will play the most important role.

Let $F: X \rightarrow Y$ be a multi-valued function and let $\alpha \in \operatorname{Cov}(X)$ and $\sigma \in \operatorname{Cov}(Y)$. We shall say that $F$ is a multi-valued $(\alpha, \sigma)$-function provided for every $A \in \alpha$ there is an $S_{A} \in \sigma$ with $F(A) \subset S_{A}$. On the other hand, $F$ is a multi-valued $\sigma$-function provided there is an $\alpha \epsilon$ $\operatorname{Cov}(X)$ such that $F$ is a multi-valued $(\alpha, \sigma)$-function.

Also important will be the following concept of closeness for two multi-valued functions.

Let $F, G: X \rightarrow Y$ be multi-valued functions, let $\sigma \in \operatorname{Cov}(Y)$, and let $\alpha \in \operatorname{Cov}(X)$. We shall say that $F$ and $G$ are $(\alpha, \sigma)$-close and we write $F \stackrel{(\alpha, \sigma)}{=} G$ provided for every $A$ in $\alpha$ there is an $S_{A} \in \sigma$ with $F(A) \cup G(A) \subset S_{A}$. 


\section{NORMAL BICOVERS AND MULTI-VALUED BIFUNC- TIONS}

In this section we shall define versions for maps of some notions from the previous section.

By a normal bicover of a bispace $X$ we mean a pair $\left(\sigma^{\prime}, \sigma\right)$, where $\sigma^{\prime}$ is a normal cover of the space $X^{\prime}$ and $\sigma$ is a normal cover of the space $X$ such that $\sigma^{\prime}$ refines the cover $x^{-1}(\sigma)$. We shall again make a simplification of our notation by dropping $\sigma^{\prime}$ so that normal bicovers are denoted by small Greek letters which name a normal cover of the codomain while the part in the domain space has the prime.

Let $\operatorname{Cov}^{b}(Y)$ denote the collection of all normal bicovers of a bispace $Y$. We order $\operatorname{Cov}^{b}(Y)$ by the refinement relation $\geq$ defined by $\sigma \geq \tau$ if and only if $\sigma \geq \tau$ and $\sigma^{\prime} \geq \tau^{\prime}$ for normal bicovers $\sigma$ and $\tau$ of $Y$. With respect to the relation $\geq$ the set $\operatorname{Cov}^{b}(Y)$ is a directed set. Two normal bicovers $\sigma$ and $\tau$ of a bispace $Y$ are equivalent provided $\sigma \geq t$ and $\tau \geq \sigma$. In order to simplify our notation we denote a normal bicover and it's equivalence class by the same symbol. Consequently, $\operatorname{Cov}^{b}(Y)$ also stands for the associated quotient set.

If $\sigma$ is a normal bicover of a bispace $Y$, let $\sigma^{+}$be the collection of all normal bicovers of $Y$ which refine $\sigma$ while $\sigma^{*}$ denotes the set of all normal bicovers $\tau$ of $Y$ such that the star $\operatorname{st}(\tau)$ of $\tau$ refines $\sigma$. Here, we define the star st $(\sigma)$ of a normal bicover $\left(\sigma^{\prime}, \sigma\right)$ as a normal bicover $\left(s t\left(\sigma^{\prime}\right), s t(\sigma)\right)$. Similarly, for a natural number $n, \sigma^{* n}$ denotes the set of all normal bicovers $\tau$ of $Y$ such that the $n$-th star $s t^{n}(\tau)$ of $\tau$ refines $\sigma$.

Let $Y$ be a bispace. Let $I n c^{b}(Y)$ denote the collection of all finite subsets $c$ of $\operatorname{Cov}^{b}(Y)$ which have a unique (with respect to the refinement relation) maximal element which we denote by $[c]$. We consider $I n c^{b}(Y)$ ordered by the inclusion relation and regard $\operatorname{Cov}^{b}(Y)$ as a subset a singleelement subsets of $\operatorname{Cov}^{b}(Y)$. Notice that $I n c^{b}(Y)$ is a cofinite directed set.

For our approach to shape theory of maps the following class of multi-valued bifunctions will play the most important role.

Let $X$ and $Y$ be bispaces. By a multi-valued bifunction from $X$ into $Y$ we mean a pair $\left(A^{\prime}, A\right)$ of multi-valued functions $A^{\prime}: X^{\prime} \rightarrow Y^{\prime}$ and $A: \quad X \rightarrow Y$. We shall drop $A^{\prime}$ and use $A$ to denote the multi-valued 
bifunction $\left(A^{\prime}, A\right)$. Of course, we shall utilize the functional notation $A: X \rightarrow Y$ to indicate that $A$ is a multi-valued bifunction between bispaces $X$ and $Y$. The multi-valued bifunctions $A$ and $B$ are composed by the rule $B \circ A=\left(B^{\prime} \circ A^{\prime}, B \circ A\right)$ and multiplied by the rule $A \times B=$ $\left(A^{\prime} \times B^{\prime}, A \times B\right)$.

Let $X$ and $Y$ be bispaces. Let $\alpha$ and $\beta$ be normal bicovers of $X$ and $Y$. By a multi-valued $(\alpha, \beta)$-bifunction from $X$ into $Y$ we mean a multivalued bifunction $A: X \rightarrow Y$ such that $A^{\prime}$ is a multi-valued $\left(\alpha^{\prime}, \beta^{\prime}\right)$ function, $A$ is a multi-valued $(\alpha, \beta)$-function and the compositions $y \circ A^{\prime}$ and $A \circ x$ are $\left(\alpha^{\prime}, \beta\right)$-close.

On the other hand, $A$ is a multi-valued $\beta$-bifunction provided there is a normal bicover $\alpha \in \operatorname{Cov}^{b}(X)$ such that $A$ is a multi-valued $(\alpha, \beta)$ bifunction.

\section{THE RELATION OF $\sigma$-BIHOMOTOPY}

Now we a ready to introduce an important notion of $\sigma$-bihomotopy for multi-valued bifunctions of bispaces. We shall also prove in Lemma 2 a useful technical result.

Let $F$ and $G$ be multi-valued bifunctions between bispaces $X$ and $Y$ and let $\sigma$ be a normal bicover of $Y$. We shall say that $F$ and $G$ are $\sigma$-bihomotopic and write $F \stackrel{\sigma}{\simeq_{b}} G$ provided there is a multi-valued $\sigma$-bifunction $H$ from the bicylinder $X \times I$ into $Y$. such that $F^{\prime}\left(a^{\prime}\right)=$ $H^{\prime}\left(a^{\prime}, 0\right)$ and $G^{\prime}\left(a^{\prime}\right)=H^{\prime}\left(a^{\prime}, 1\right)$ for every $a^{\prime} \in X^{\prime}$ and $F(a)=H(a, 0)$ and $G(a)=H(a, 1)$ for every $a \in X$. We shall say that $H$ is a $\sigma$ bihomotopy that joins $F$ and $G$ or that it realizes the relation (or $\sigma$ bihomotopy) $F \stackrel{\sigma}{\simeq_{b}} G$.

The following lemma is crucial because it provides an adequate substitute for the transitivity of the relation of $\sigma$-bihomotopy.

Lemma 2. Let $F, G$, and $H$ be multi-valued bifunctions between bispaces $X$ and $Y$. Let $\sigma$ be a normal bicover of $Y$ and let $\tau \in \sigma^{*}$. If

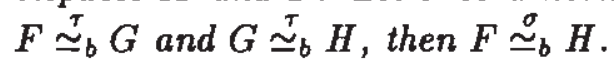

Proof. By assumption there are normal bicovers $\alpha$ and $\beta$ of the bicylinder $X \times I$, a multi-valued $(\alpha, \tau)$-bifunction $K: X \times I \rightarrow Y$, and a multi-valued $(\beta, \tau)$-bifunction $L: X \times I \rightarrow Y^{\prime}$ so that $F^{\prime}\left(a^{\prime}\right)=$ 
$K^{\prime \prime}\left(a^{\prime}, 0\right), G^{\prime}\left(a^{\prime}\right)=K^{\prime}\left(a^{\prime}, 1\right)=L^{\prime}\left(a^{\prime} ; 0\right)$, and $H^{\prime}\left(a^{\prime}\right)=L^{\prime}\left(a^{\prime}, 1\right)$, for every $a^{\prime} \in X^{\prime}$, and $F(a)=K(a, 0), G(a)=K(a, 1), G(a)=L(a, 0)$, and $L(a, 1)=H(a)$ for every $a \in X$. Let a normal bicover $\gamma$ of $X \times I$ be a common refinement of $\alpha$ and $\beta$. Observe first that $K$ and $L$ are both multi-valued $(\gamma, \tau)$-bifunctions. Define a multi-valued bifunction $M: X \times I \rightarrow Y$ by the rule

$$
M^{\prime}\left(a^{\prime}, t\right)= \begin{cases}K^{\prime}\left(a^{\prime}, 2 t\right), & a^{\prime} \in X^{\prime}, 0 \leq t \leq 1 / 2 \\ L^{\prime}\left(a^{\prime}, 2 t-1\right), & a^{\prime} \in X^{\prime}, 1 / 2 \leq t \leq 1\end{cases}
$$

and

$$
M(a, t)= \begin{cases}K(a, 2 t), & a \in X, 0 \leq t \leq 1 / 2 \\ L(a, 2 t-1), & a \in X, 1 / 2 \leq t \leq 1,\end{cases}
$$

Since $F^{\prime}\left(a^{\prime}\right)=M^{\prime}\left(a^{\prime}, 0\right)$ and $H^{\prime}\left(a^{\prime}\right)=M^{\prime}\left(a^{\prime}, 1\right)$ for every $a^{\prime} \in X^{\prime}$, and $F(a)=M(a, 0)$ and $H(a)=M(a, 1)$ for every $a \in X$, it remains to see that $M$ is a multi-valued $\sigma$-bifunction.

By $\left[5\right.$, p. 358], there are normal covers $\delta^{\prime}$ and $\delta$ of $X^{\prime}$ and $X$ and functions $r^{\prime}: \delta^{\prime} \rightarrow\{2,3,4, \ldots\}$ and $r: \delta \rightarrow\{2,3,4, \ldots\}$ such that the set $D^{\prime} \times D_{i}^{\prime}$ is contained in a member $C_{D^{\prime}, i}^{\prime}$ of $\gamma^{\prime}$ for every $D^{\prime} \in \delta^{\prime}$ and every $i=1, \ldots, r^{\prime} D^{\prime}-1$, where $D_{i}^{\prime}=\left[\frac{i-1}{r^{\prime} D^{\prime}}, \frac{i+1}{C^{\prime}}\right]\left(i=1, \ldots, r^{\prime} D^{\prime}-1\right)$ and the set $D \times D_{i}$ is contained in a member $C_{D, i}$ of $\gamma$ for every $D \in \delta$ and every $i=1, \ldots, r D-1$, where $D_{i}=\left[\frac{i-1}{r D}, \frac{i+1}{r D}\right](i=1, \ldots, r D-1)$.

We can assume that $\delta^{\prime}$ refines $x^{-1}(\delta)$. Then for every $D^{\prime} \in \delta^{\prime}$ there is a $D\left(D^{\prime}\right) \in \delta$ such that $D^{\prime}$ is a subset of $x^{-1}\left(D\left(D^{\prime}\right)\right)$. Let $s D^{\prime}=\left(r^{\prime} D^{\prime}\right)\left(r D\left(D^{\prime}\right)\right)$ for every $D^{\prime} \in \delta^{\prime}$ and let

$$
E_{i}= \begin{cases}{\left[0, \frac{1}{2 r D}\right),} & i=0, \\ \left(\frac{i}{4 r D}, \frac{i+2}{4 r D}\right), & i=1, \ldots, 4 r D-3, \\ \left(1-\frac{1}{2 r D}, 1\right], & i=4 r D-2\end{cases}
$$


and similarly

$$
E_{i}^{\prime}= \begin{cases}{\left[0, \frac{1}{2 s D^{\prime}}\right),} & i=0, \\ \left(\frac{i}{4 s D^{\prime}}, \frac{i+2}{2 s D^{\prime}}\right), & i=1, \ldots, 4 s D^{\prime}-3, \\ \left(1-\frac{1}{2 s D^{\prime}}, 1\right], & i=4 s D^{\prime}-2 .\end{cases}
$$

The collections

$$
\varepsilon^{\prime}=\left\{D^{\prime} \times E_{i}^{\prime} \mid D^{\prime} \in \delta^{\prime}, i=0, \ldots, 4 s D^{\prime}-2\right\}
$$

and

$$
\varepsilon=\left\{D \times E_{i} \mid D \in \delta, i=0,1, \ldots, 4 r D-2\right\}
$$

are normal covers of $X^{\prime} \times I$ and $X \times I$. Moreover, we claim that the pair $\left(\varepsilon^{\prime}, \varepsilon\right)$ is a normal bicover of the bicylinder $X \times I$.

Indeed, let $T=D^{\prime} \times E_{i}^{\prime}$ be a member of $\varepsilon^{\prime}$. Since the set $R=$ $D \times E$, where $D=D\left(D^{\prime}\right)$ and $E=E_{j}$, is a member of $\varepsilon$ for every $j=0,1, \ldots 4 r E-2$, the preimage $\left(x \times 1_{I}\right)^{-1}(R)$ is a set $x^{-1}(D) \times E$, and $D^{\prime} \subset x^{-1}(D)$, it suffices to show that there exists an index $j$ such that $E_{i}^{\prime} \subset E_{j}$.

When $i=0$, then we can take $j=0$ because

$$
E_{0}^{\prime}=\left[0, \frac{1}{2 s D^{\prime}}\right)=\left[0, \frac{1}{2 T^{\prime} D^{\prime} D\left(D^{\prime}\right)}\right) \subset\left[0, \frac{1}{2 r D}\right)=E_{0} .
$$

For a similar reason, when $i=4 s D^{\prime}-2$, then we can take $j=4 r D-2$. Finally, when $i \in\left\{1, \ldots, 4 s D^{\prime}-3\right\}$, the required inclusion will hold provided $\frac{i}{4 s D^{\prime}} \geq \frac{j}{4 r D}$ and $\frac{i+2}{4 s D^{\prime}} \leq \frac{j+2}{4+D}$. Thus, the index $j$ must satisfy conditions $\frac{i+2}{r^{\prime} D^{\prime}}-2 \leq j \leq \frac{i}{r^{\prime} D^{\prime}}$. But, if we write $i=r^{\prime} D^{\prime} m+n$, for integers $m$ and $n$ with $0 \leq n<r^{\prime} D^{\prime}$, then these conditions become $j \geq m-2+\frac{n+2}{\frac{r^{\prime} D^{\prime}}{}}$ and $j \leq m+\frac{n}{r^{\prime} D^{\prime}}$. It is clear that $j=m$ always satisfies them. Another possibility is $j=m-1$ in some situations. Therefore, $E_{i}^{\prime} \subset E_{m}^{\prime}$ and our claim has been verified. 
In order to prove that $M$ is a multi-valued $\sigma$-bifunction, we shall show that it is a multi-valued $(\varepsilon, \sigma)$-bifunction. In other words, we shall prove that

(1) $M^{\prime}$ is a multi-valuéd $\left(\varepsilon^{\prime}, \sigma^{\prime}\right)$-function,

(2) $M$ is a multi-valued $(\varepsilon, \sigma)$-function, and

(3) the compositions $y \circ\left(M^{\prime}\right)$ and $M \circ\left(x \times 1_{I}\right)$ are $\left(\varepsilon^{\prime}, \sigma\right)$-close.

Add (1). The argument for (1) is similar to the argument for (2) given below. Of course, the case (2) is notationally simpler because there are no primes.

Add (2). Let $E=D \times E_{i}$ be a member of $\varepsilon$. We must find an $S_{E} \in \sigma$ such that $M(E) \subset S_{E}$.

Case I ( $i=2 k$ for $0 \leq k \leq r D-1)$. Then $E_{i}=E_{2 k}$ is below $1 / 2$ so that we get $M(E)=K\left(D \times 2 E_{i}\right)=K\left(D \times D_{k}\right) \subset K\left(C_{D, k}\right) \subset T \subset S_{E}$, where the open set $T$ is a member of $T$ which we obtain with respect to $C_{D, k}$ from the fact that $K$ is a multi-valued $(\gamma, \tau)$-function and $S_{E}$ is a member of $\sigma$ which contains $T$.

Case II $(i=2 k$ for $r D \leq k \leq 2 r D-1)$. Then $E_{i}=E_{2 k}$ is above $1 / 2$ so that we get $M(E)=L\left(D \times\left(2 E_{i}-1\right)\right) \subset L\left(D \times D_{k \rightarrow r D}\right) \subset$ $L\left(C_{D, k-r D}\right) \subset T \subset S_{E}$, where the open set $T$ is a member of $\tau$ which we obtain with respect to $C_{D, k-\tau D}$ from the fact that $L$ is a multi-valued $(\gamma, \tau)$-function, and $S_{E}$ is a member of $\sigma$ which contains the set $T$.

Case III $(i=2 k+1$ for $0 \leq k \leq r D-2)$. Then $E_{i}=E_{2 k+1}$ is below $1 / 2$ so that we get

$$
\begin{aligned}
M(E)=K\left(D \times 2 E_{i}\right) & \subset K\left(D \times\left(D_{k} \cup D_{k+1}\right)\right) \\
& \subset K\left(C_{D, k}\right) \cup K\left(C_{D, k+1}\right) \subset T_{1} \cup T_{2} \subset S_{E},
\end{aligned}
$$

where the sets $T_{1}$ and $T_{2}$ are members of $\tau$ which we obtain with respect to $C_{D, k}$ and $C_{D, k+1}$ from the fact that $K$ is a $(\gamma, \tau)$-function, and $S_{E}$ is a member of $\sigma$ which contains the union $T_{1} \cup T_{2}$. Such an $S_{E}$ exists because $D \times\{k / r D\} \subset C_{D, k} \cap C_{D, k+1}$ so that $\emptyset \neq K(D \times\{k / r D\}) \subset T_{1} \cap T_{2}$.

Case IV ( $i=2 k+1$ for $r D \leq k \leq 2 r D-1)$. This case is analogous to the case III. This time $E_{i}$ is above $1 / 2$ and we must use $L$ instead of $K$. 
Case $\mathrm{V}(i=2 k+1$ and $k=r D-1)$. Then $E_{\mathrm{i}}=E^{-1} \cup E^{+}$, where $E^{-}=\left(\frac{2 r D-1}{4 r D}, \frac{1}{2}\right]$ and $E^{+}=\left[\frac{1}{2}, \frac{2 r D+1}{4}\right)$ so that we get

$$
\begin{gathered}
M(E)=K\left(D \times 2 E^{-}\right) \cup L\left(D \times\left(2 E^{+}-1\right)\right) \subset K\left(C_{D, r D-1}\right) \cup L\left(C_{D, 1}\right) \\
\subset T_{1} \cup T_{2},
\end{gathered}
$$

where $T_{1}$ and $T_{2}$ are members of $\tau$ which we obtain with respect to $C_{D, r D-1}$ and $C_{D, 1}$ from the fact that $K$ and $L$ are multi-valued $(\gamma, \tau)$ functions.

Observe that $K(D \times\{1\})=G(D) \subset T_{1}$ and $L(D \times\{0\})=G(D) \subset$ $T_{2}$. We conclude that $T_{1} \cap T_{2} \neq \emptyset$ so that there is a member $S_{E}$ of $\sigma$ which contains both $T_{1}$ and $T_{2}$. It follows that $M(E) \subset S_{E}$.

Add (3). Let $E^{\prime}=D^{\prime} \times E_{i}^{\prime}$ be a member of $\varepsilon^{\prime}$. We must find a member $S_{E^{\prime}}$ of $\sigma$ such that both $M \circ\left(x \times 1_{I}\right)\left(E^{\prime}\right)$ and $y \circ\left(M^{\prime}\right)\left(E^{\prime}\right)$ are contained in the set $S_{E^{\prime}}$.

Once again we shall distinguish five cases considered above.

Case I ( $i=2 k$ for $0 \leq k \leq r^{\prime} D^{\prime}-1$ ). Let $A^{\prime}$ be a member of $\alpha^{\prime}$ which contains the set $C_{D^{\prime}, k}^{\prime}$. Since $K \circ\left(x \times 1_{I}\right)$ and $y \circ\left(K^{\prime}\right)$ are $\left(\alpha^{\prime}, \tau\right)$ close, there is a member $T$ of $\tau$ which contains both $K \circ\left(x \times 1_{I}\right)\left(A^{\prime}\right)$ and $y \circ\left(K^{\prime}\right)\left(A^{\prime}\right)$. Since $\tau$ is a star-refinement of $\sigma$, there is an $S_{E} \in \sigma$ with $T \subset S_{E}$. Our choices imply

$$
y \circ\left(M^{\prime}\right)\left(E^{\prime}\right) \subset y \circ\left(K^{\prime}\right)\left(C_{D^{\prime}, k}^{\prime}\right) \subset y \circ\left(K^{\prime}\right)\left(A^{\prime}\right)
$$

and

$$
\begin{aligned}
M \circ\left(x \times 1_{I}\right)\left(E^{\prime}\right) & \subset M\left(x\left(D^{\prime}\right) \times E_{i}^{\prime}\right)=K\left(x\left(D^{\prime}\right) \times 2 E_{i}^{\prime}\right) \\
& \subset K \circ\left(x \times 1_{I}\right)\left(D^{\prime} \times D_{k}^{\prime}\right) \subset K \circ\left(x \times 1_{I}\right)\left(A^{\prime}\right)
\end{aligned}
$$

because $E^{\prime} \subset C_{D^{\prime}, k}^{\prime} \subset A^{\prime}$. It follows that $M \circ\left(x \times 1_{I}\right)\left(E^{\prime}\right)$ and $y \circ$ $\left(M^{\prime}\right)\left(E^{\prime}\right)$ are both subsets of $S_{E^{\prime}}$.

Case II ( $i=2 k$ for $\left.r^{\prime} D^{\prime} \leq k \leq 2 r^{\prime} D^{\prime}-1\right)$. This is a similar to the previous case. We have to deal with $L$ instead of with $K$.

Case III $\left(i=2 k+1\right.$ for $\left.0 \leq k \leq r^{\prime} D^{\prime}-2\right)$. The set $y \circ\left(M^{\prime}\right)\left(E^{\prime}\right)$ is now a subset of the union of sets $y \circ\left(K^{\prime}\right)\left(C_{D^{\prime}, k}^{\prime}\right)$ and $y \circ\left(K^{\prime}\right)\left(C_{D^{\prime}, k+1}^{\prime}\right)$. 
Let $\delta A_{1}^{\prime}$, and $\lrcorner A_{2}^{\prime-}$ be members of $\alpha^{\prime}$ which contain $C_{D^{\prime}, k}^{\prime}$ and $C_{D^{\prime}, k+1}^{\prime}$, respectively. Since, $K \circ\left(x \times 1_{I}\right)$ and $y \circ\left(K^{\prime}\right)$ are $\left(\alpha^{\prime}, \tau\right)$-close, there are members $T_{1}$ and $T_{2}$ of $\tau$ such that $T_{1}$ contains $K \circ\left(x \times 1_{I}\right)\left(A_{1}^{\prime}\right)$ and $y \circ\left(K^{\prime}\right)\left(A_{1}^{\prime}\right)$ while $T_{2}$ contains $K \circ\left(x \times 1_{I}\right)\left(A_{2}^{\prime}\right)$ and $y \circ\left(K^{\prime}\right)\left(A_{2}^{\prime}\right)$. The sets $T_{1}$ and $T_{2}$ both contain the set $y \circ\left(K^{\prime}\right)\left(D^{\prime} \times\left\{\frac{k}{r^{\prime} D^{\prime}}\right\}\right)$. It follows that some member $S_{E^{\prime}}$ of $\sigma$ contains their union. This is the required open set.

of therdacis ntiph

$(T$, Case IV This is similar to the previous case.

Case V. The set $y \circ\left(M^{\prime}\right)\left(E^{\prime}\right)$ is a subset of the union of sets $y \circ$ $\left(K_{0}^{\prime}\right)\left(C_{D^{\prime} ; r^{\prime} D^{\prime}, \Omega_{1}^{\prime}}^{\prime}\right)$ and $y \circ\left(L^{\prime}\right)\left(C_{D^{\prime}, 1}^{\prime}\right)$. Let $A_{1}^{\prime}$ be a member of $\alpha^{\prime}$ and let $B_{2}^{\prime}$ be a member of $\beta^{\prime}$ such that $A_{1}^{\prime}$ contains $C_{D^{\prime}, \tau^{\prime} D^{\prime}-1}^{\prime}$ and $B_{2}^{\prime}$ contains $C_{D^{\prime}, 1}^{\prime}$. Since $K \circ\left(x \times 1_{I}\right)$ is $\left(\alpha^{\prime}, \tau\right)$-close to $y \circ\left(K^{\prime}\right)$ and $L \circ\left(x \times 1_{I}\right)$ is $\left(\beta^{\prime}, \tau\right)$ close to $y \circ\left(L^{\prime}\right)$, there are members $T_{1}$ and $T_{2}$ of $\tau$ such that $T_{1}$ contains $\hat{K} \quad b\left(x^{\prime} x^{\prime} 1_{I}^{\prime}\right)\left(A_{1}^{\prime}\right)$ and $y \circ\left(K^{\prime}\right)\left(A_{1}^{\prime}\right)$ while $T_{2}$ contains $L \circ\left(x \times 1_{I}\right)\left(B_{2}^{\prime}\right)$ and $y^{15}{ }^{5}\left(L^{\prime}\right)\left({ }^{\prime} B_{2}^{\prime}\right) .{ }^{\prime}$ But the sets $T_{1}$ and $T_{2}$ both contain the non-empty set

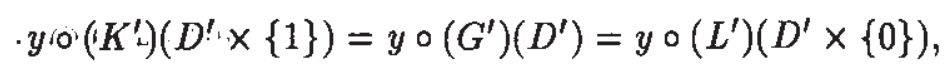

'o to rodmon is ...

so- that some'member $S_{E^{\prime}}$ of $\sigma$ contains their union. It follows that $M_{k \circ}\left(x_{1} \mid X_{1}\right)\left(E_{i}^{i}\right)$ and $y \circ\left(M^{\prime}\right)\left(E^{\prime}\right)$ are both subsets of $S_{E^{\prime}}$.

- ${ }^{\exists}$ Since ${ }^{r}$ we shâll be using $[5$, p. 358] quite often, for a space $Y$ and a normal cover $\alpha$ of the product $Y \times I$, we let $D(Y, \alpha)$ denote all normal covers $\beta$ of $Y$ such that some stacked normal cover over $\beta$ refines $\alpha$.

$$
\text { ( h): }
$$

\section{MULTI-BINETS}

The following two definitions correspond to Borsuk's definitions of fundamental sequence and homotopy for fundamental sequences [3].

Let $X$ and $Y$ be bispaces. By a multi-binet from $X$ into $Y$ we shall mean a collection $\varphi=\left\{F_{c} \mid c \in \operatorname{Inc}(Y)\right\}$ of multi-valued bifunctions $F_{c}: X \rightarrow Y$ such that for every $\sigma \in \operatorname{Cov}^{b}(Y)$ there is a $c \in \operatorname{Inc}^{b}(Y)$ with ${ }^{b} \simeq_{b} F_{c}$ for every $d \geq c$. We use functional notation $\varphi: X \rightarrow Y$ to indicate that $\varphi$ is a multi-binet from $X$ into $Y$. Let $M^{b}(X, Y)$ denote all multi:binets: $\varphi: X \rightarrow Y$ from $X$ into $Y$.

Multi-binets $\varphi=\left\{F_{c}\right\}$ and $\psi=\left\{G_{c}\right\}$ between bispaces $X$ and $Y$ are 'said lto be bihomotopic and we write $\varphi \simeq_{b} \psi$ provided for every $\sigma \in C o^{\text {irb }}(Y)$ there is a $c \in \operatorname{Inc}(Y)$ such that $F_{d} \stackrel{\sigma}{\sim_{b}} G_{d}$ for every $d \geq c$. 
It follows from Lemma 2 that the relation of bihomotopy is:an equivalence relation on the set $M^{b}(X, Y)$. The bihomotopy class of a multi-binet $\varphi$ is denoted by $[\varphi]_{b}$ and the set of all bihomotopy classes by $\mathcal{H} M^{b}(X, Y)$.

\section{COMPOSITION OF BIHOMOTOPY CLASSES OF MULTI-BINETS}

Our principal goal now is to define a composition for bihomotopy classes of multi-binets and to establish it's associativity. This is the only tricky part in setting up the category $\mathcal{H} M^{b}$. Our idea is to associate to every multi-binet $\varphi: X \rightarrow Y$ two increasing functions $\varphi: \operatorname{Inc} c^{b}(Y) \rightarrow \operatorname{In} c^{b}(Y)$ and $\varphi^{*}: \operatorname{Inc} c^{b}(Y) \rightarrow \operatorname{In} c^{b}(X)$. The first function associates to an index $c \in \operatorname{Inc} c^{b}(Y)$ of the family $\varphi^{\prime}=\left\{F_{c}^{\prime}\right\}$ a much larger index $\varphi(c)$ in $I n c^{b}(Y)$ such that $F_{d}$ and $F_{c}$ are joined by a multi-valued $(\varphi(c, d, e),[c])$-bihomotopy for a suitable normal bicover $\varphi(c, d, e)$ of the bicylinder $X \times I$ whenever $d, e \geq \varphi(c)$. The second function associates to a $c \in \operatorname{In} c^{b}(Y)$ an element $\varphi^{*}(c)$ of $I n c^{b}(X)$ such that the normal bicover $\left[\varphi^{*}(c)\right]$ refines some normal bicover of $X$ which we get from the normal bicover $\varphi(c, \varphi(c), \varphi(c))$ by [5, p. 358]. This is a rough description of these functions and now we proceed with the details.

Let $X$ and $Y$ be bispaces. Let $\varphi=\left\{F_{c}\right\}_{c \in \operatorname{Inc}(Y)}: X \rightarrow Y$ be a multi-binet. Let $\varphi: I n c^{b}(Y) \rightarrow I n c^{b}(Y)$ be an increasing function such that for every $c \in \operatorname{Inc}^{b}(Y)$ the relation $d, e \geq \varphi(c)$ implies the relation $F_{d} \stackrel{[c]}{\stackrel{[}{\sim}} F_{e}$, where $[c]$ denotes the unique maximal element of $c$.

of

Let $\mathcal{C}_{\varphi}=\left\{(c, d, e) \mid c \in \operatorname{Inc}^{b}(Y), d, e \geq \varphi(c)\right\}$. Then $\mathcal{C}_{\varphi}^{\prime}$ is a subset

$$
\operatorname{Inc} c^{b}(Y) \times \operatorname{In} c^{b}(Y) \times \operatorname{Inc} c^{b}(Y)
$$

that becomes a cofinite directed set when we define that $(c, d, e) \geq$ $\left(c^{\prime}, d^{\prime}, e^{\prime}\right)$ if and only if $c \geq c^{\prime}, d \geq d^{\prime}$, and $e \geq e^{\prime}$ for $(c, d, e)$ and $\left(c^{\prime}, d^{\prime}, e^{\prime}\right)$ in $\mathcal{C}_{\varphi}$.

We shall use the same notation $\varphi$ for an increasing function $\varphi$ : $\mathcal{C}_{\varphi} \rightarrow \operatorname{Cov}^{b}(X \times I)$ such that $F_{d}$ and $F_{e}$ are joined by a multi-valued $(\varphi(c, d, e),[c])$-bihomotopy whenever $(c, d, e) \in \mathcal{C}_{\varphi}$.

Let $\bar{\varphi}: \mathcal{C}_{\varphi} \rightarrow \operatorname{Inc}(X)$ be an increasing function such that the normal cover $\left[(\bar{\varphi}(c, d, e))^{\prime}\right]$ belongs to the set $D\left(X,(\varphi(c, d, e))^{\prime}\right)$ and the 
normal cover $[\bar{\varphi}(c, d, e)]$ belongs to the set $D(X, \varphi(c, d, e))$ for every $(c, d, e) \in \mathcal{C}_{\varphi}$. such that

Claim 1. There is an increasing function $\varphi^{*}: \operatorname{Inc}(Y) \rightarrow \operatorname{Inc}(X)$

(1) $\varphi^{*}(c) \geq \bar{\varphi}(c, \varphi(c), \varphi(c))$ for every $c \in \operatorname{Inc}(Y)$, and

(2) $\varphi^{*}$ is cofinal in $\bar{\varphi}$, i.e., for every $(c, d, e) \in C_{\varphi}$ there is an $m \in \operatorname{Inc} c^{b}(Y)$ with $\varphi^{*}(m) \geq \bar{\varphi}(c, d, e)$.

Proof. Let $\mathcal{D}=\left\{\bar{\varphi}(c, d, e) \mid(c, d, e) \in \mathcal{C}_{\varphi}\right\}$.

If $I n c^{b}(Y)$ is a finite set, then $\mathcal{D}$ is a finite collection of elements of $I n c^{b}(X)$. Let $a \in I n c^{b}(X)$ be greater than all members of $\mathcal{D}$. Let $\varphi^{*}: \operatorname{Inc}(Y) \rightarrow \operatorname{Inc} c^{b}(X)$ be a constant function into $a$.

If $I n c^{b}(Y)$ is an infinite set, then the cardinality of $\mathcal{D}$ does not exceed the cardinality of $\operatorname{Inc}(Y)$. Hence, there is a surjection $g: \operatorname{Inc} c^{b}(Y)$ $\rightarrow \mathcal{D}$. Let $\varphi^{*}: \operatorname{Inc} c^{b}(Y) \rightarrow \operatorname{Inc}(X)$ be an increasing function such that $\varphi^{*}(c) \geq g(c), \bar{\varphi}(c, \varphi(c), \varphi(c))$ for every $c \in I n c^{b}(Y)$.

The above discussion shows that every multi-binet $\varphi: X \rightarrow Y$ determines two increasing functions $\varphi: I n c^{b}(Y) \rightarrow I n c^{b}(Y)$ and $\varphi^{*}$ : $I n c^{b}(Y) \rightarrow I n c^{b}(X)$. With the help of these functions we shall define the composition of bihomotopy classes of multi-binets as follows.

Let $X, Y$, and $Z$ be bispaces and let $\varphi=\left\{F_{c}\right\}: X \rightarrow Y$ and $\psi=\left\{G_{s}\right\}: Y \rightarrow Z$ be multi-binets. Let $\chi=\left\{H_{s}\right\}$, where $H_{s}=$ $G_{\psi_{(s)}} \circ F_{\varphi\left(\psi^{-}(s)\right)}$ for every $s \in I n c^{b}(Z)$. Observe that each $H_{s}$ is a multi-valued bifunction because the composition of two multi-valued bifunctions is a multi-valued bifunction.

Claim 2. The collection $\chi$ is a multi-binet from $X$ into $Z$.

Proof. Let $\sigma \in \operatorname{Cov}^{b}(Z)$. We must find a $u \in \operatorname{Inc} c^{b}(Z)$ such that

$$
H_{v} \stackrel{g}{\simeq} H_{u} \quad \text { for every } v \geq u
$$

Let $\tau \in \sigma^{* 2}$ and $\xi \in \tau^{*}$. Let $u=\{\xi\} \in I n c^{b}(Z)$. 
Consider an index $v \geq u$. We shall find an index $e \in I n c^{b}(Y)$ so that

$$
\begin{gathered}
H_{v} \stackrel{\tau}{\simeq}_{b} G_{q} \circ F_{e}, \\
G_{q} \circ F_{e} \stackrel{\tau}{\simeq}_{b} G_{p} \circ F_{e}
\end{gathered}
$$

and

$$
G_{p} \circ F_{e} \stackrel{\tau}{\simeq}_{b} H_{u},
$$

where $p=\psi(u), q=\psi(v), a=\psi^{*}(u), b=\psi^{*}(v), c=\varphi(a)$, and $d=\varphi(b)$. Repeated use of Lemma 2 will give (3) from the relations (4)-(6).

Add (4). Since $(v, q, q) \in \mathcal{C}_{\varphi}$, we see that there is a multi-valued $(\alpha, \xi)$-bihomotopy $K: Y \times I \rightarrow Z$ such that $\alpha=\psi(v, y, y), K_{0}^{\prime}=$ $G_{q}^{\prime}, K_{1}^{\prime}=G_{q}^{\prime}, K_{0}=G_{q}$, and $K_{1}=G_{q}$. Let $s=\bar{\psi}(v, q, q)$ and $\pi=[s]$. Observe that $\pi^{\prime} \in D\left(Y^{\prime}, \alpha^{\prime}\right)$ and $\pi \in D(Y, \alpha)$. We claim that $G_{q}$ is a multi-valued $(\pi, \xi)$-bifunction from $Y$ into $Z$. In other words, we must show that

(a) $G_{q}^{\prime}$ is a multi-valued $\left(\pi^{\prime}, \xi^{\prime}\right)$-function,

(b) $G_{q}$ is a multi-valued $(\pi, \xi)$-function,

(c) the compositions $z \circ G_{q}^{\prime}$ and $G_{q} \circ y$ are $\left(\pi^{\prime}, \xi\right)$-close.

Add (a). Let $P^{\prime}$ be a member of $\pi^{\prime}$. Then there is a $t \geq 0$ such that the product $P^{\prime} \times[0, t)$ lies in a member $A^{\prime}$ of $\alpha^{\prime}$. Since $K^{\prime}$ is an $\left(\alpha^{\prime}, \xi^{\prime}\right)$-function, there is a $T^{\prime} \in \xi^{\prime}$ such that $K^{\prime}\left(A^{\prime}\right) \subset T^{\prime}$. It follows that $G_{q}^{\prime}\left(P^{\prime}\right)=K_{0}^{\prime}\left(P^{\prime}\right)=K^{\prime}\left(P^{\prime} \times\{0\}\right) \subset K^{\prime}\left(A^{\prime}\right) \subset T^{\prime}$ which proves that $G_{q}^{\prime}$ is a $\left(\pi^{\prime}, \xi^{\prime}\right)$-function. above.

Add (b). The proof of (b) is analogous to the proof for (a) given

Add (c). Since the compositions $K \circ\left(y \times 1_{I}\right)$ and $z \circ\left(K^{\prime}\right)$ are $\left(\alpha^{\prime}, \xi\right)$ close so that their restrictions $\left.K \circ\left(y \times 1_{I}\right)\right|_{Y^{\prime} \times\{0\}}$ and $\left.z \circ\left(K^{\prime}\right)\right|_{Y^{\prime} \times\{0\}}$ on $Y^{\prime} \times\{0\}$ will be $\left(\left.\alpha^{\prime}\right|_{Y^{\prime} \times\{0\}}, \xi\right)$-close. But, if we identify the space $Y^{\prime}$ with $Y^{\prime} \times\{0\}$ in $Y^{\prime} \times I$ we see that the normal cover $\pi^{\prime}$ refines $\left.\alpha^{\prime}\right|_{Y^{\prime}}$, and that the restrictions above are $y \circ G_{q}$ and $z \circ G_{q}^{\prime}$. Hence, $z \circ G_{q}^{\prime}$ and $y \circ G_{q}$ are $\left(\pi^{\prime}, \xi\right)$-close.

Once we know that $G_{q}$ is a multi-valued $(\pi, \xi)$-bifunction, we see that it suffices to take $e \geq d$ because then $F_{d}$ and $F_{e}$ are joined by 
anniulti:valued $(\varepsilon,[b])$-bihomotopy $L: X \times I \rightarrow Y$, for some normal bicover $\varepsilon$ of $X \times I$, so that $G_{q} \circ L$ is a multi-valued $(\varepsilon, \tau)$-bihomotopy which realizes the relation (4).

(c) Indeed, by construction, the normal bicover $[b]$ refines $\pi$ (i.e., $[b]^{\prime}$ refines $\pi^{\prime}$ and $[b]$ refines $\pi$ ) so that $G_{q}^{\prime} \circ L^{\prime}$ is a multi-valued $\left(\varepsilon^{\prime}, \xi^{\prime}\right)$ function and $G_{q} \circ L$ is a multi-valued $(\varepsilon, \xi)$-function. On the other hand, since the compositions $y \circ\left(L^{\prime}\right)$ and $L \circ\left(x \times 1_{I}\right)$ are $\left(\varepsilon^{\prime},[b]\right)$-close, the function $G_{q}$ is a multi-valued $(\pi, \xi)$-bifunction, and the normal cover $[b]$ refines $\pi$, it follows that $G_{q} \circ y \circ\left(L^{\prime}\right)$ and $G_{q} \circ L \circ\left(x \times 1_{I}\right)$ are $\left(\varepsilon^{\prime}, \xi\right)$ close. But, the compositions $G_{q} \circ y$ and $z \circ G_{q}^{\prime}$ are $\left(\pi^{\prime}, \xi\right)$-close, $L^{\prime}$ is a multi-valued $\left(\varepsilon^{\prime},[b]^{\prime}\right)$-bifunction, and the normal cover $[b]^{t}$ refines $\pi^{\prime}$ so that $G_{q}+y \circ\left(L^{\prime}\right)$ and $z \circ G_{q}^{\prime} \circ\left(L^{\prime}\right)$ are $\left(\varepsilon^{\prime}, \xi\right)$-close. Since the cover $\operatorname{st}(\xi)$ refines the cover $\tau$, we conclude that the compositions $z \circ G_{q}^{\prime} \circ\left(L^{\prime}\right)$ and $G_{q} \circ L^{\prime \prime} \circ\left(x x^{\prime} 1_{I}\right)$ are $\left(\varepsilon^{\prime}, \tau\right)$-close.

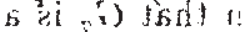

jerri Add (5) Since $(u, p, q) \in \mathcal{C}_{\psi}$, it follows that $G_{p}$ and $G_{q}$ are joined by a multi-valued $(\alpha, \xi)$-bihomotopy $K: Y \times I \rightarrow Z$, where $\alpha$ denotes the normal bicover $\psi(u, p, q)$ of $Y \times I$. Choose normal covers $\beta^{\prime}$ of $Y^{\prime}$ and $\beta$ of $Y$ and functions $r^{\prime}: \beta^{\prime} \rightarrow\{2,3,4, \ldots\}$ and $r: \beta \rightarrow\{2,3,4, \ldots\}$ such that sets $B^{\prime} \times\left[\frac{i-1}{r^{\prime} B^{\prime}}, \frac{i+1}{r^{\prime} B^{\prime}}\right]$ and $B \times\left[\frac{j-1}{r B}, \frac{j+1}{r B}\right]$, where $B^{\prime} \in \beta^{\prime}, B \in$ $\beta, i=1, \ldots, r^{\prime} B^{\prime}-1$, and $j=1, \ldots, r B-1$ are contained in a member $A_{B^{\prime}, i}^{\prime}$ of $\alpha^{\prime}$ and in a member $A_{B, j}$ of $\alpha$, respectively. We can assume that $\beta^{\prime}$ refines $y^{-1}(\beta)$. It follows that a pair $\left(\beta^{\prime}, \beta\right)$ is a normal bicover of the' bispace' $Y$. Let $k=\{\beta\}$ and $e=\varphi(k)$. Since $(k, e, e) \in \mathcal{C}_{\varphi}$, the

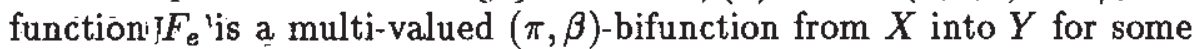
normal bicover $\pi$ of $X$. It follows that for every $P^{\prime} \in \pi^{\prime}$, every $P \in \pi$, and every $B^{\prime} \in \beta^{\prime}$ there is a $B_{P^{\prime}}^{\prime}$ from $\beta^{\prime}$ and a $\left[B^{\prime}\right]$, a $B_{P^{\prime}}$, and a $B_{P}$ in $\beta$ such that $B^{\prime} \subset y^{-1}\left(\left[B^{\prime}\right]\right), F_{e}^{\prime}\left(P^{\prime}\right) \subset B_{P^{\prime}}^{\prime}, F_{e}(P) \subset B_{P}$, and $B_{P^{\prime}}$ contains both $y$ o $F_{e}^{\prime}\left(P^{\prime}\right)$ and $F_{e} \circ x\left(P^{\prime}\right)$.

$$
\begin{aligned}
& \text { For every } P^{\prime} \in \pi^{\prime} \text { and every } P \in \pi \text {, let } s^{\prime} P^{\prime}=r^{\prime} B_{P^{\prime}}^{\prime} r B_{P^{\prime}} r\left[B_{P^{\prime}}^{\prime}\right] \text {, } \\
& \text {. } \\
& \text { bith ivos men } \\
& 4 \text { are } \\
& \bigcup\left\{\left(\frac{i}{s^{\prime} P^{\prime}}, \frac{i+2}{s^{\prime} P^{\prime}}\right) \mid i=1, \ldots, s^{\prime} P^{\prime}-3\right\} \\
& \text { यो batgited on }
\end{aligned}
$$


and

$$
\begin{aligned}
\nu_{P}= & \left\{\left[0, \frac{2}{r \dot{B}_{P}}\right),\left(\frac{r B_{P}-2}{r B_{P}}, 1\right]\right\} \\
& \bigcup\left\{\left(\frac{i}{r B_{P}}, \frac{i+2}{r B_{P}}\right) \mid i=1, \ldots, r B_{P}-3\right\} .
\end{aligned}
$$

Put $\varrho^{\prime}=\left\{P^{\prime} \times N^{\prime} \mid P^{\prime} \in \pi^{\prime}, N^{\prime} \in \nu_{P^{\prime}}^{\prime}\right\}$ and $\varrho=\{P \times N \mid P \in \pi, N \in$ $\left.\nu_{P}\right\}$. Observe that $\varrho^{\prime}$ and $\varrho$ are normal covers of $X^{\prime} \times I$ and $X \times I$, respectively. Let $\omega$ be a normal bicover of $X \times I$ such that the normal cover $\omega^{\prime}$ refines $\varrho^{\prime}$ and the normal cover $\omega$ refines $\varrho$. We claim that the composition $H=K \circ\left(F_{e} \times 1_{I}\right)$ is a multi-valued $(\omega, \tau)$-bihomotopy which joins $G_{p} \circ F_{e}$ and $G_{q} \circ F_{e}$. Thus, we must show that

(a) $H^{\prime}$ is a multi-valued $\left(\omega^{\prime}, \tau^{\prime}\right)$-function,

(b) $H$ is a multi-valued $(\omega, \tau)$-function, and

(c) the compositions $z \circ H^{\prime}$ and $H \circ\left(x \times 1_{I}\right)$ are $\left(\omega^{\prime}, \tau^{\prime}\right)$-close.

Add (a). Let $S^{\prime}$ be a member of $\omega^{\prime}$. Pick a member $R^{\prime}=P^{\prime} \times N^{\prime}$ of $\varrho^{\prime}$ which contains $S^{\prime}$, where $P^{\prime} \in \pi^{\prime}$ and $N^{\prime} \in \nu_{P}^{\prime}$. Then ' .

$-H^{\prime}\left(S^{\prime}\right) \subset H^{\prime}\left(R^{\prime}\right)=K^{\prime}\left(F_{e}^{\prime}\left(P^{\prime}\right) \times N^{\prime}\right) \subset K\left(B_{p}^{\prime} \times N^{\prime}\right) \subset K^{\prime}\left(A_{B_{p \prime}^{\prime}, j}^{\prime}\right)$,

where $j$ is such that $N^{\prime}$ lies is the interior of the segment $\left[\frac{1}{N_{p^{\prime}}^{\prime}}, \frac{j+2}{\gamma^{\prime} B_{p^{\prime}}^{\prime}}\right]$. Such an index can surely be found because the lenght of $N^{\prime}$ is less than $\frac{1}{T^{\prime} B_{p^{\prime}}^{r}}$. Since $K^{\prime}$ is a multi-valued $\left(\alpha^{\prime}, \xi^{\prime}\right)$-homotopy, we obtain that the last set in the chain of inclusions above is a subset of a member of $\xi^{\prime}$. Hence, $H^{\prime}$ is a multi-valued $\left(\omega^{\prime}, \tau^{\prime}\right)$-function.

Add (b). The proof of (b) is analogous to the proof of (a).

Add (c). Let $S^{\prime}$ be a member of $\omega^{\prime}$. Choose sets $P^{\prime}, N^{\prime}$, and $R^{\prime}$ as above. Observe that the sets $y \circ F_{e}^{\prime}\left(P^{\prime}\right)$ and $F_{e} \circ x\left(P^{\prime}\right)$ both lie in a member $B_{P^{\prime}}$ of $\beta$. Since the length of the segment $N^{\prime}$ is less than $\frac{1}{r B_{P^{\prime}}}$, the product $B_{P^{\prime}} \times N^{\prime}$ lies in a member $A_{B_{P^{\prime}, j}}$ of $\alpha$, for some $j \in\left\{1, \ldots, r B_{P^{\prime}}-1\right\}$. This implies that some member $T_{1}$ of $\xi$ contains sets $A=K\left(y \circ F_{e}^{\prime}\left(P^{\prime}\right) \times N^{\prime}\right)$ and $B=K\left(F_{e} \circ x\left(P^{\prime}\right) \times N^{\prime}\right)$ because $K$ is a multi-valued $(\alpha, \xi)$-function. Notice that $B=K \circ\left(F_{e} \times 1_{I}\right) \circ\left(x \times 1_{I}\right)\left(R^{\prime}\right)$. On the other hand, the inclusions $F_{e}^{\prime}\left(P^{\prime}\right) \subset B_{P^{\prime}}^{\prime}$ and $B_{P^{\prime}}^{\prime} \subset y^{-1}\left(\left[B_{P^{\prime}}^{\prime}\right]\right)$ 
imply that $y \circ F_{e}^{\prime}\left(P^{\prime}\right) \times N^{\prime} \subset\left[B_{P^{\prime}}^{\prime}\right] \times N^{\prime}$ and therefore that $A$ is a subset of the set $D=K\left(\left[B_{P^{\prime}}^{\prime}\right] \times N^{\prime}\right)$. Moreover, since the length of $N^{\prime}$ is less than $\frac{1}{p_{p^{\prime}}^{\prime}}$ the product $L=\left[B_{P^{\prime}}^{\prime}\right] \times N^{\prime}$ lies in a member $A_{\left[B_{P^{\prime}}^{\prime}\right], k}$ of $\alpha$ for some $k \in\left\{1, \ldots, r\left[B_{P^{\prime}}^{\prime}\right]-1\right\}$. Hence, some member $T_{2}$ of $\xi$ contains the set $K(L)$. The inclusion $y\left(B_{P^{\prime}}^{\prime}\right) \subset\left[B_{P^{\prime}}^{\prime}\right]$ now gives that $J=K \circ\left(y \times 1_{I}\right)\left(B_{P^{\prime}}^{\prime} \times N^{\prime}\right)$ is a subset of $K(L)$. Once again, since the length of $N^{\prime}$ is less than $\frac{1}{r^{\prime} B_{P^{\prime}}^{\prime}}$, the set $B_{P^{\prime}}^{\prime} \times N^{\prime}$ lies in a member $E^{\prime}=A_{B_{p}^{\prime}, m}^{\prime}$ of $\alpha^{\prime}$ for some $m \in\left\{1, \ldots, r^{\prime} B_{P^{\prime}}^{\prime}\right\}$. We conclude that $J$ is a subset of $K \circ\left(y \times 1_{I}\right)\left(E^{\prime}\right)$. Now we use the assumption that $z \circ\left(K^{\prime}\right)$ and $K \circ\left(y \times 1_{I}\right)$ are $\left(\alpha^{\prime}, \xi\right)$-close to find a member $T_{3}$ of $\xi$ which contains the sets $K \circ\left(y \times 1_{I}\right)\left(E^{\prime}\right)$ and $z \circ\left(K^{\prime}\right)\left(E^{\prime}\right)$. It remains to observe that the inclusion $F_{e}^{\prime}\left(P^{\prime}\right) \subset B_{P}^{\prime}$ implies

$$
\begin{aligned}
z \circ\left(K^{\prime}\right) \circ\left(F_{e}^{\prime} \times 1_{I}\right)\left(R^{\prime}\right)= & z \circ\left(K^{\prime}\right)\left(F_{e}^{\prime}\left(P^{\prime}\right) \times N^{\prime}\right) \subset \\
& z \circ\left(K^{\prime}\right)\left(B_{P}^{\prime} \times N^{\prime}\right) \subset z \circ\left(K^{\prime}\right)\left(E^{\prime}\right) .
\end{aligned}
$$

Finally, it follows that the star of $T_{2}$ with respect to $\xi$, and therefore also some member of $\tau$, contains sets $z \circ\left(K^{\prime}\right) \circ\left(F_{e}^{\prime} \times 1_{I}\right)\left(S^{\prime}\right)$ and $K \circ$ $\left(F_{e} \times 1_{I}\right) \circ\left(x \times 1_{I}\right)\left(S^{\prime}\right)$.

Add (6). The proof of (6) is analogous to the proof of (4).

We now define the composition of bihomotopy classes of multibinets by the rule $\left[\left\{G_{s}\right\}\right] \circ\left[\left\{F_{c}\right\}\right]=\left[\left\{G_{\psi(s)} \circ F_{\varphi\left(\psi^{*}(s)\right)}\right\}\right]$.

Claim 3. The composition of bihomotopy classes of multi-binets is well-defined.

Proof. Let $\kappa=\left\{K_{c}\right\}$ and $\lambda=\left\{L_{s}\right\}$ be multi-binets bihomotopic to $\varphi$ and $\psi$, respectively, and let $\mu=\left\{M_{s}\right\}$, where $M_{s}=L_{\lambda(s)} \circ K_{\kappa\left(\lambda^{*}(s)\right)}$ for every $s \in \operatorname{Inc}^{b}(Z)$. We must show that multi-binets $\chi$ and $\mu$ are bihomotopic. In other words, that for every $\sigma \in \operatorname{Cov}^{b}(Z)$ there is an $s \in \operatorname{Inc} c^{b}(Z)$ such that

$$
H_{t} \stackrel{\sigma}{\simeq}_{b} M_{t} \text { for every } t \geq s
$$


Let $\sigma \in \operatorname{Cov}^{b}(Z)$. Let $\tau \in \sigma^{* 4}$ and $\xi \in \tau^{*}$. Let $s=\{\xi\} \in \operatorname{Inc}(Z)$. In order to prove (7), we shall argue that for every $t \geq s$ we can find indices $e \in I n c^{b}(Y)$ and $u \in I n c^{b}(Z)$ such that

$$
\begin{gathered}
H_{t} \stackrel{\tau}{\simeq}_{b} G_{p} \circ F_{e}, \\
G_{p} \circ F_{e} \stackrel{\tau}{\simeq}_{b} G_{u} \circ F_{e}, \\
G_{u} \circ F_{e} \stackrel{\tau}{\simeq}_{b} L_{u} \circ F_{e}, \\
L_{u} \circ F_{e} \stackrel{\tau}{\simeq}_{b} L_{u} \circ K_{e}, \\
L_{u} \circ K_{e} \stackrel{\tau}{\simeq}_{b} L_{q} \circ K_{e}, \\
L_{q} \circ K_{e} \stackrel{\tau}{\simeq_{b}} M_{t},
\end{gathered}
$$

where we put $p=\psi(t), a=\psi^{*}(t), b=\varphi(a), q=\lambda(t), c=\lambda^{*}(t)$, and $d=\kappa(c)$. From the relations (8) $-(13)$ with the help of Lemma 2 we shall get (7).

We shall now describe how big $e$ and $u$ must be chosen for relations (8), (9), (10), and (11) to hold separately. The relations (12) and (13) are analogous to relations (9) and (8), respectively. We leave to the reader the task of making a cumulative choice for $e$ and $u$ which accomplishes our goal. It is important to notice that $u$ is selected first while $e$ is selected only once $u$ is already known.

Add (8). We know from the proof of Claim 2 that $G_{p}$ is a multivalued $(\pi, \xi)$-bifunction from the bispace $Y$ into the bispace $Z$, where $\pi=[s]$ and $s=\bar{\psi}(t, p, p)$. Since $a \geq s$ by the property (1) of Claim 1, it suffices to take $e \geq b$.

Add (9). If $u \geq p$, then $G_{p}$ and $G_{u}$ are joined by a multi-valued $(\alpha, \xi)$-bihomotopy $K: Y \times I \rightarrow Z$, where $\alpha=\bar{\psi}(u, m, m)$ and $m=\psi(u)$. Choose normal covers $\beta^{\prime}$ of $Y^{\prime}$ and $\beta$ of $Y$ and functions $r^{\prime}: \beta^{\prime} \rightarrow$ $\{2,3,4, \ldots\}$ and $r: \beta \rightarrow\{2,3,4, \ldots\}$ such that the sets $B^{\prime} \times\left[\frac{i-1}{\tau^{\prime} B^{\prime}}, \frac{i+1}{\tau^{\prime} B^{\prime}}\right]$ and $B \times\left[\frac{j-1}{r B}, \frac{j+1}{r B}\right]$, where $B^{\prime} \in \beta^{\prime}, B \in \beta, i=1, \ldots, r^{\prime} B^{\prime}-1$, and $j=1, \ldots, r B-1$, are contained in a member $A_{B^{\prime}, i}^{\prime}$ of $\alpha^{\prime}$ and in a member $A_{B, j}$ of $\alpha$, respectively. We can assume that $\beta^{\prime}$ refines $y^{-1}(\beta)$ so that the pair $\left(\beta^{\prime}, \beta\right)$ is a normal bicover of $Y$. Let $k=\{\beta\}$ and $e \geq \varphi(k)$. Just as in the proof of (5) we can see that $K \circ\left(F_{e} \times 1_{I}\right)$ is 
a multi-valued $\tau$-bihomotopy joining the left and the right side of the relation (9).

Add (10). Since multi-binets $\psi$ and $\lambda$ are bihomotopic, there is a $u \in \operatorname{Inc} c^{b}(Z)$, a normal bicover $\alpha$ of $Y \times I$, and a multi-valued $(\alpha, \xi)$ bihomotopy $S: Y \times I \rightarrow Z$ joining $G_{u}$ and $L_{u}$. Choose a normal bicover $\beta$ and an index $e$ as above. Then $S \circ\left(F_{e} \times 1_{I}\right)$ is a multi-valued $\tau$-bihomotopy joining compositions which appear in (10).

Add (11). Let $u \geq q$. Then $L_{u}$ is a multi-valued $(\alpha, \xi)$-bifunction from $Y$ into $Z$, where $\alpha=[s], s=\bar{\lambda}(u, w, w)$, and $w=\lambda(u)$. Since multi-binets $\varphi$ and $k$ are bihomotopic, there is an index $e \in I n c^{b}(Y)$ so that $F_{e}$ and $K_{e}$ are joined by a multi-valued $\alpha$-bihomotopy $T: X \times I \rightarrow$ $Y$. The composition $L_{u} \circ T$ realizes the relation (11).

Theorem 1. The composition of bihomotopy classes of multi-binets is associative.

Proof. Let $\varphi=\left\{F_{c}\right\}, \psi=\left\{G_{s}\right\}$, and $\chi=\left\{H_{p}\right\}$ be multi-binets from $X$ into $Y$, from $Y$ into $Z$, and from $Z$ into $W$, respectively. Let $\mu=$ $\left\{M_{s}\right\}, \nu=\left\{N_{p}\right\}, \kappa=\left\{K_{p}\right\}$, and $\lambda=\left\{L_{p}\right\}$, where $M_{s}=G_{\psi(s)} \circ F_{\varphi\left(\psi^{*}(s)\right)}$ for every $s \in I n c^{b}(Z)$ and $N_{p}=H_{\chi(p)} \circ G_{\psi\left(\chi^{*}(p)\right)}, K_{p}=H_{\chi(p)} \circ M_{\mu\left(\chi^{*}(p)\right)}$, and $L_{p}=N_{\nu(p)} \circ F_{\varphi\left(\nu^{*}(p)\right)}$, for every $p \in \operatorname{Inc} c^{b}(W)$. We must show that multi-binets $\kappa$ and $\lambda$ are bihomotopic, i.e., that for every normal bicover $\pi \in \operatorname{Cov}^{b}(W)$ there is an index $p \in \operatorname{Inc}^{b}(W)$ such that

$$
K_{q} \stackrel{\pi}{\simeq}_{b} L_{q} \quad \text { for every } q \geq p
$$

Let $\pi \in \operatorname{Cov}^{b}(W)$. Let $\varrho \in \pi^{* 4}, \xi \in \varrho^{*}$, and $\eta \in \xi^{*}$. Let $p=\{\eta\} \in$ $I n c^{b}(W)$. In order to prove (14), we shall show that for every $q \geq p$ we can find indices $e \in I n c^{b}(Y)$ and $s \in \operatorname{Inc} c^{b}(Z)$ such that

$$
\begin{aligned}
& K_{q} \stackrel{\varrho}{\simeq}_{b} H_{m} \circ G_{n} \circ F_{e}, \\
& H_{m} \circ G_{n} \circ F_{e} \stackrel{Q}{\simeq}_{b} H_{m} \circ G_{s} \circ F_{e}, \\
& H_{m} \circ G_{s} \circ F_{e} \stackrel{\varrho}{\simeq} H_{k} \circ G_{s} \circ F_{e},
\end{aligned}
$$




$$
H_{k} \circ G_{s} \circ F_{\mathrm{e}} \stackrel{e}{ }_{b} N_{l} \circ F_{e}
$$

and

$$
N_{l} \circ F_{e} \stackrel{\varrho}{=} L_{q},
$$

where $m=\chi(q), n=\psi\left(\mu\left(\chi^{*}(q)\right)\right), k=\chi(\nu(q))$, and $l=\nu(q)$. Repeated use of Lemma 2 will give (14) from the relations (15)-(19).

The method of proof is similar to the proof of Claim 3. We shall only describe for each of the relations (15)-(19) how large the indices $u$ and $e$ must be in order that this multi-valued $\varrho$-bihomotopy holds. An easy exercise of putting together all these selections is once again left to the reader. Since relations (18) and (19) are analagous with relations (16) and (15), respectively, it suffices to consider only relations (15)-(17).

Add (15). Observe that $H_{m}$ is a multi-valued $(\theta, \eta)$-bifunction, where $\theta=[a], a=\bar{\chi}(q, m, m)$. Let $i=\chi^{*}(q), j=\mu(i), h=\psi(j), d=$ $\bar{\psi}(j, n, n), c=\psi^{*}(j)$, and $\omega=[d]$. Then $G_{n}$ is a multi-valued $(\omega,[j])$ bifunction from $Y$ into $Z$. Since $j \geq i$ and $i \geq a$ by the property (1) from Claim 1, we obtain that $[j]$ refines $\theta$. Let $b=\varphi(c)$. If $e \geq b$, then $F_{e}$ and $F_{b}$ are joined by a multi-valued [c]-bihomotopy $P$. But, $c \geq d$ so that $[c]$ refines $\omega$. Hence, $H_{m} \circ G_{n} \circ P$ is a multi-valued $\varrho$-bihomotopy between $K_{q}$ and $H_{m} \circ G_{n} \circ F_{e}$.

Add (16). As above, $H_{m}$ is a multi-valued $(\theta, \eta)$-bifunction from $Z$ into $W$. If we take $s>n$, then $(j, n, s) \in \mathcal{C}_{\psi}$ so that $G_{n}$ and $G_{s}$ are joined by a multi-valued $(\alpha,[j])$-bihomotopy $Q: ' Y \times I \rightarrow Z$, where $\alpha=\psi(j, n, s)$. But, since $i \geq a$, we see that $[n]$ refines $\theta$. Choose normal covers $\beta^{\prime}$ of $Y^{\prime}$ and $\beta$ of $Y$ and functions $r^{\prime}: \beta^{\prime} \rightarrow\{2,3,4, \ldots\}$ and $r: \beta \rightarrow\{2,3,4, \ldots$,$\} such that sets B^{\prime} \times\left[\frac{i-1}{r^{\prime} B^{\prime}}, \frac{i+1}{r^{\prime} B^{\prime}}\right]$ and $B \times\left[\frac{i-1}{r B}, \frac{i+1}{r B}\right]$, where $B^{\prime} \in \beta^{\prime}, B \in \beta, i=1, \ldots, r^{\prime} B^{\prime}-1$, and $j=1, \ldots, r B-1$, are contained in a member $A_{B^{\prime}, i}^{\prime}$ of $\alpha^{\prime}$ and in a member $A_{B, j}$ of $\alpha$, respectively. We can assume that $\beta^{\prime}$ refines $y^{-1}(\beta)$ so that the pair $\left(\beta^{\prime}, \beta\right)$ is a normal bicover for the bispace $Y$. Let $g=\{\beta\}$ and $e \geq \varphi(g)$. Just as in the proofs of (5) and (9), we can see that $H_{m} \circ Q \circ\left(F_{e} \times 1_{I}\right)$ realizes the relation (16).

Add (17). Since $\nu(r) \geq r$ for every $r \in \operatorname{Inc}(W)$, we get $k \geq m$ so that $(q, m, k) \in \mathcal{C}_{\chi}$ and $H_{m}$ and $H_{k}$ are joined by a multi-valued $(\alpha, \eta)$ bihomotopy $T: Z \times I \rightarrow W$, where $\alpha=\chi(q, m, k)$. Let $v=\chi^{*}(k)$ and let $s \geq \psi(v)$. Then $G_{s}$ is a multi-valued $([t],[v])$-bifunction from $Y$ into 
$Z$, where $t=\bar{\psi}(s, \psi(s), \psi(s))$. Let $u=\psi^{*}(t)$ and take $e \geq \varphi(u)$. The composition $T \circ\left(\left(G_{s} \circ F_{e}\right) \times 1_{I}\right)$ realizes the relation (17).

\section{THE SHAPE CATEGORY OF MAPS $\mathcal{H} M^{b}$}

For a topological bispace $X$, let $i^{X}=\left\{I_{a}\right\}: X \rightarrow X$ be the identity multi-binet defined by $I_{a}=1_{X}$ for every $a \in \operatorname{Inc} c^{b}(X)$. It is easy to show that for every multi-binet $\varphi: X \rightarrow Y$, the following relations hold:

$$
[\varphi]_{b} \circ\left[i^{X}\right]_{b}=[\varphi]_{b}=\left[i^{Y}\right]_{b} \circ[\varphi]_{b}
$$

We can summarize the above with the following theorem which is the main result of this paper.

Theorem 2. The topological bispaces as objects together with the bihomotopy classes of multi-binets as morphisms and the composition of bihomotopy classes form the category $\mathcal{H M}^{b}$.

There are some natural functors into the category $\mathcal{H} M^{b}$. First of all, there is a functor $S^{b}: \mathcal{S}^{b} \rightarrow \mathcal{H} M^{b}$ from the category $\mathcal{S}^{b}$ of bispaces and bimaps which leaves the objects unchanged and associates to a bimap $f: X \rightarrow Y$ between bispaces $X$ and $Y$ the bihomotopy class $[\varphi]_{b}$ of the multi-binet $\varphi=\left\{F_{c}\right\}: X \rightarrow Y$ generated by the bimap $f$, where $F_{c}=f$ for every $c \in \operatorname{In} c^{b}(Y)$. Then, there is an analogous functor $S_{h}^{b}: \mathcal{H}^{b} \rightarrow \mathcal{H} M^{b}$ from the category $\mathcal{H}^{b}$ of bispaces and bihomotopy classes of bimaps. Finally, there is a functor $M^{b}: \mathcal{H} M \rightarrow \mathcal{H} M^{b}$ from the author's shape category [4] of spaces and homotopy classes of multi-nets which associates to a topological space $X^{\prime}$ a bispace $x: X^{\prime} \rightarrow X$, where $X$ is a fixed single-element space, and which associates to a homotopy class $\left[\varphi^{\prime}\right]$ of a multi-net $\varphi^{\prime}: X^{\prime} \rightarrow Y^{\prime}$ between topological spaces $X^{\prime}$ and $Y^{\prime}$ the bihomotopy class $[\varphi]_{b}$ of the multi-binet $\varphi: X \rightarrow Y$ whose domains part is the multi-net $\varphi^{\prime}$. The functor $M^{b}$ is a full embedding. Since in [4] the author proved that the category $\mathcal{H} M$ is isomorphic to the shape category $S h$, we get the following corollary.

Corollary 1. Topological spaces $X$ and $Y$ have the same shape if and only if the maps of $X$ and $Y$ into a single-element space are equivalent in the category $\mathcal{H} M^{b}$. 


\section{SPECIAL MULTI-BINETS}

In this section we shall be looking for conditions under which a given bihomotopy class of multi-binets has a representative of a special kind. As a corollary of these results we identify a class of bispaces on which bishape theory and bihomotopy theory coincide.

A bispace $X$ is internally movable provided for every $\sigma \in \operatorname{Cov}^{b}(X)$ there is a $\tau \in \operatorname{Cov}^{b}(X)$ such that every multi-valued $\tau$-bifunction into $X$ is $\sigma$-bihomotopic to a bimap.

A bispace $X$ is internally calm provided there is a $\sigma \in \operatorname{Cov}^{b}(X)$ such that bimaps into $X$ which are $\sigma$-bihomotopic are bihomotopic.

A bispace $X$ is calm provided there is a $\sigma \in \operatorname{Cov}^{b}(X)$ such that for every $\tau$ in $\operatorname{Cov}^{b}(X)$ there is a $\varrho$ in $\operatorname{Cov}^{b}(X)$ with the property that multi-valued $\varrho$-bifunctions into $X$ which are $\sigma$-bihomotopic are also $\tau$ bihomotopic.

A multi-binet $\varphi=\left\{F_{c}\right\}_{c \in I n c^{b}(Y)}$ from a bispace $X$ into a bispace $Y$ is regular provided each bifunction $F_{c}$ is a bimap. It is called simple when there is a bimap $f$ such that $f=F_{c}$ for every $c \in \operatorname{In} c^{b}(Y)$.

Theorem 3. If a bispace $Y$ is internally movable, then every multibinet $\varphi$ from a bispace $X$ into $Y$ is bihomotopic to a regular multi-binet.

Proof. Since $Y$ is internally movable, for every $c \in I n c^{b}(Y)$ there is a $\chi(c) \in[c]^{+}$such that every multi-valued $\chi(c)$-bifunction into $Y$ is $[c]-$ bihomotopic to a bimap. Let $\sigma_{c} \in \chi(c)^{*}$. Let $\lambda: \operatorname{Inc} c^{b}(Y) \rightarrow \operatorname{In} c^{b}(Y)$ be an increasing function such that $\lambda(c) \geq \varphi\left(\left\{\sigma_{c}\right\}\right)$ for every $c \in \operatorname{Inc}^{b}(Y)$. Then $F_{\lambda(c)}$ is a multi-valued $\chi(c)$-bifunction so that we can select a bimap $g_{c}: X \rightarrow Y$ with $g_{c} \stackrel{\left[\stackrel{[c]}{\simeq}_{b}\right.}{ } F_{\lambda(c)}$, for every $c \in \operatorname{In} c^{b}(Y)$.

In order to verify that $\psi=\left\{g_{c}\right\}_{c \in \operatorname{Inc} c^{b}(Y)}$ is a multi-binet from $X$ into $Y$, let a $\sigma \in \operatorname{Cov}^{b}(Y)$ be given. Let $\mu \in \sigma^{*}$ and put $c=\{\mu\}$. For

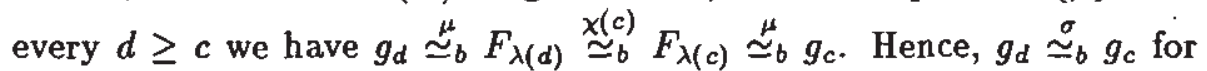
every $d \geq c$.

It remains to check that multi-binets $\varphi$ and $\psi$ are bihomotopic. Let a normal bicover $\sigma \in \operatorname{Cov}^{b}(Y)$ be given. Let $\mu \in \sigma^{*}$. Choose an index $c_{0} \in \operatorname{Inc} c^{b}(Y)$ such that $F_{d^{a}} \stackrel{\leftrightarrow}{\simeq} F_{e}$ for all $d, e \geq c_{0}$. Let $c \geq c_{0},\{\mu\}$. 
For every $d \geq c$, we get $g_{d} \stackrel{\Perp^{\prime}}{{ }_{b}} F_{\lambda(d)}$ by construction, while $F_{\lambda(d)} \stackrel{\stackrel{\mu}{\simeq}_{b}}{F_{d}}$ because $\lambda(d) \geq d \geq c_{0}$. Hence, $g_{d} \stackrel{\sigma}{\simeq_{b}} F_{d}$ for every $d \geq c$.

Theorem 4. If a bispace $Y$ is both internally movable and calm, then every multi-binet $\varphi$ into $Y$ is bihomotopic to a simple multi-binet.

Proof. Since $Y$ is calm, there is a normal bicover $\gamma \in \operatorname{Cov}^{b}(Y)$ such that for every $\sigma \in \operatorname{Cov}^{b}(Y)$ there is a $\tau \in \operatorname{Cov}^{b}(Y)$ with the property that $\gamma$-bihomotopic multi-valued $\tau$-bifunctions into $Y$ are in fact $\sigma$-bihomotopic.

Let $\delta \in \gamma^{*}$. Since the bispace $Y$ is also internally movable, there is an $\eta \in \delta^{+}$such that multi-valued $\eta$-bifunctions into $Y$ are $\delta$-bihomotopic to bimaps. Let $c=\varphi(\{\eta\})$. Then $F_{c}$ is a multi-valued $\eta$-bifunction so that it is $\delta$-bihomotopic to a bimap $g$. Let $\psi$ denote the simple multibinet determined by the bimap $g$.

In order to check that $\varphi$ and $\psi$ are bihomotopic, let a normal bicover $\sigma$ of $Y$ be given. Choose a $\tau \in \operatorname{Cov}^{b}(Y)$ as above. Since $\varphi$ is a multibinet, there is an index $d \geq c$ such that $F_{e}$ is a multi-valued $\tau$-bifunction for every $e \geq d$. Thus, for every $e \geq d$ we get $F_{e} \stackrel{\beth}{\sim}_{b} F_{c} \stackrel{\delta}{\simeq} g$. Hence, $F_{e} \stackrel{\gamma}{\simeq}_{b} g$ so that $F_{e} \stackrel{g}{\simeq}_{b} g$.

Theorem 5. Let $Y$ be a internally calm bispace and let $\varphi=\{f\}$ and $\psi=\{g\}$ be simple multi-binets into $Y$. If $\varphi$ and $\psi$ are bihomotopic, then the bimaps $f$ and $g$ are bihomotopic.

Proof. Since the bispace $Y$ is internally calm, there is a $\sigma \in$ $\operatorname{Cov}^{b}(Y)$ such that $\sigma$-bihomotopic bimaps into $Y$ are in fact bihomotopic. But, the assumption that the multi-binets $\varphi$ and $\psi$ are bihomotopic gives $f \stackrel{\sigma}{\simeq_{b}} g$. Hence, the bimaps $f$ and $g$ are bihomotopic.

The last three theorems imply that the functor $S_{h}^{b}$ is an isomorphism of categories when we restrict to bispaces that have the above properties.

Let $\mathcal{A}$ denote the collection of all bispaces that are at the same time internally movable, internally calm, and calm.

Let $\mathcal{H}_{A}^{b}$ be the full subcategory of $\mathcal{H}^{b}$ with objects precisely the members of the collection $\mathcal{A}$. The category $\mathcal{H} M_{\mathcal{A}}^{b}$ is deftned similarly. 
Let $T_{h}^{b}: \mathcal{H}_{\mathcal{A}}^{b} \rightarrow \mathcal{H} M_{\mathcal{A}}^{b}$ be the restriction of the functor $S_{h}^{b}$ to the category $\mathcal{H}_{\mathcal{A}}^{b}$.

Theorem 6. The functor $T_{h}^{b}$ is an isomorphism of categories.

Proof. We shall construct a functor $K_{h}^{b}: \mathcal{H} M_{A}^{b} \rightarrow \mathcal{H}_{A}^{b}$ which satisfies the relations $T_{h}^{b} \circ K_{h}^{b}=I d$ and $K_{h}^{b} \circ T_{h}^{b}=I d$. The functor $K_{h}^{b}$ leaves the objects unchanged and on morphisms it is defined as follows. Let $C$ be a bihomotopy class of multi-binets between two members $X$ and $Y$ of $\mathcal{A}$. Let $\varphi$ be a representative of $C$ and let $g: X \rightarrow Y$ be a bimap such that the simple multi-binet $\psi$ determined by $g$ is bihomotopic to $\varphi$. The functor $K_{h}^{b}$ associates to $C$ the bihomotopy class of the bimap $g$. It follows from the above results that this definition is correct and that $K_{h}^{b}$ has the required properties.

Corollary 2. On bispaces which are at the same time internally movable, calm, and internally calm, the bihomotopy theory and the bishape theory coincide.

\section{CHARACTERIZATION OF BISHAPE EQUIVALENCES}

In this section we shall use classes of internally movable and internally calm bispaces to obtain an analogue for bimaps of $G$. Kozlowski's characterization of shape equivalences in terms of induced functions of homotopy classes.

For bispaces $X$ and $Y$, let $[X, Y]_{b}$ denote the set of all bihomotopy classes of bimaps from $X$ into $Y$. Every bimap $f: X \rightarrow Y$ induces for every bispace $Z$ a set function $f_{Z}^{\#}:[Y, Z]_{b} \rightarrow[X, Z]_{b}$ defined by the rule $f_{X}^{\#}\left([a]_{b}\right)=[a \circ f]_{b}$, for every bimap $a: Y \rightarrow Z$.

Recall that a morphism $\varphi$ of the bishape category $\mathcal{H} M^{b}$ is bishape left invertible provided there is another bishape morphism $\psi$ with the composition $\psi \circ \varphi$ equal to the identity morphism. The bishape right invertible morphisms (or shortly bishape dominations) are defined similarly. A bishape morphism is a bishape equivalence iff it is both bishape left invertible and bishape right invertible. When applied to bimaps this notions mean that the bishape morphism determined by this bimap has them. 
The following four theorems are similar to the characterization of shape equivalences from [7]. We get information on conditions which imply that functions $f_{Z}^{\#}$ induced by a bimap $f: X \rightarrow Y$ and a bispace $Z$ are surjections and injections and we get an information on what kind of bispaces $Z$ one can use.

Theorem 7. If a bimap $f: X \rightarrow Y$ between topological bispaces is bishape left invertible, then for every bispace $Z$ which is at the same time internally movable and internally calm the induced function $f_{Z}^{\#}$ is a surjection.

Proof. Let $h: X \rightarrow Z$ be a bimap. The assumption about the bispace $Z$ imply the existence of normal bicovers $\sigma \in \operatorname{Cov}^{b}(Z), \tau \in \sigma^{*}$, and $\mu \in \tau^{+}$such that $\sigma$-bihomotopic bimaps into $Z$ are bihomotopic and every multi-valued $\mu$-bifunction into $Z$ is $\tau$-bihomotopic to a bimap. Let $\varrho=h^{-1}(\mu)$. Observe that $\varrho$ is a normal bicover of $X$. Since $f$ is bishape left invertible, there is a multi-binet $\psi: Y \rightarrow X$ with $\psi \circ f$ bihomotopic to the identity multi-binet on $X$. In particular, there is an index $a \geq\{\varrho\}$ in $I n c^{b}(X)$ such that $G_{p} \circ f \stackrel{\mathscr{Q}}{\stackrel{b}{b}_{b}} 1_{X}$, where $p=\psi(a)$. Let $d=\psi^{*}(a)$.

The bifunction $G_{p}$ is a multi-valued ([d], $\varrho$ )-bifunction so that there is a bimap $k: Y \rightarrow Z$ which is $\tau$-bihomotopic to the composition $h \circ G_{p}$. It follows that $k$ of and $h$ are $\sigma$-bihomotopic and therefore bihomotopic. In other words, $f_{Z}^{\#}\left([k]_{b}\right)=[h]_{b}$.

Theorem 8. If a bimap $f: X \rightarrow Y$ between topological bispaces is bishape right invertible, then for every internally calm bispace $Z$ the induced function $f_{Z}^{\#}$ is an injection.

Proof. Let $h, k: Y \rightarrow Z$ be bimaps and assume that $f_{Z}^{\#}\left([h]_{b}\right)=$ $f_{Z}^{\#}\left([k]_{b}\right)$, i.e., that $h \circ f$ and $k \circ f$ are joined by a bihomotopy $m$ : $X \times Y \rightarrow Z$. Since $Z$ is internally calm, there is a $\sigma \in \operatorname{Cov}^{b}(Z)$ such that $\sigma$-bihomotopic bimaps into $Z$ are bihomotopic. Let $\mu \in \sigma^{*}$. Let $\varrho$ be a common refinement of $h^{-1}(\mu)$ and $k^{-1}(\mu)$. Let $\pi \in \varrho^{*}$. Since $f$ is a bishape domination, there is a multi-binet $\psi: Y \rightarrow X$ with the composition $f \circ \psi$ bihomotopic to the identity multi-binet on $Y$. Hence, there is a multi-valued $\pi$-bihomotopic joining $1_{Y}$ and $f \circ G_{p}$, where $\lambda=f^{-1}(\pi), n=\{\lambda\}$, and $p=\psi(n)$. 
Let $\alpha \in \operatorname{Cov}^{b}(X)$ be such that $\alpha^{\prime} \in D\left(X^{\prime},\left(m^{\prime}\right)^{-1}\left(\varrho^{\prime}\right)\right)$ and $\alpha \in$ $D\left(X, m^{-1}(\varrho)\right)$. Pick an index $q \geq p$ such that $G_{q}$ is a multi-valued $\alpha$-bifunction. Then $G_{q}{\stackrel{\lambda}{\sim_{b}}}_{b} G_{p}$ so that

$$
f \circ G_{q} \stackrel{\pi}{\sim}_{b} f \circ G_{p} \stackrel{\pi}{\simeq}_{b} 1_{Y}
$$

It follows that $h \circ f \circ G_{q} \stackrel{\mu}{\sim}_{b} h$ and similarly $k \circ f \circ G_{q} \stackrel{\mu}{\sim} b$. On the other hand $m \circ\left(G_{q} \times 1_{I}\right)$ is a multi-valued $\mu$-bihomotopy joining $h \circ f \circ G_{q}$ and $k \circ f \circ G_{q}$. Hence, $h \stackrel{\sigma}{\simeq_{b}} k$ and therefore $h \simeq_{b} k$.

In order to formulate a partial converse to theorem 7 and for some other results below we shall need the following notion.

Let $\mathcal{P}$ be a class of bispaces. By a $\mathcal{P}$-exposition of a bispace $X$ we mean a family $\left\{X_{a}\right\}_{a \in I n c^{b}(X)}$ of members of $\mathcal{P}$, a family $\left\{p_{b}^{a} \mid a, b \in\right.$ $\left.I n c^{b}(X), a \leq b\right\}$ of bimaps $p_{b}^{a}: X_{b} \rightarrow X_{a}$, a family $\left\{p^{a} \mid a \in I n c^{b}(X)\right\}$ of bimaps $p^{a}: X \rightarrow X_{a}$, and a family $\left\{J_{a} \mid a \in I n c^{b}(X)\right\}$ of multi-valued [a]-bifunctions such that

(1) The relation $a \leq b$ in $I n c^{b}(X)$ implies $p^{a} \simeq_{b} p_{b}^{a} \circ p^{b}$,

(2) For every $a \in \operatorname{Inc} c^{b}(X)$, we have $J_{a} \circ p^{a} \stackrel{[a]}{\simeq}_{b} 1_{X}$, and

(3) The relation $a \leq b$ in $I n c^{b}(X)$ implies $J_{b} \stackrel{[a]}{\simeq} J_{a} \circ p_{b}^{a}$.

Theorem 9. Let $\mathcal{P}$ be a class a bispaces. If a bispace $X$ has a $\mathcal{P}$-exposition and a bimap $f: X \rightarrow Y$ is such that the induced function $f_{Z}^{\#}$ is a bijection for every $Z \in \mathcal{P}$, then $f$ is bishape left invertible.

Proof. By assumption the bispace $X$ has a $\mathcal{P}$-exposition formed by bispaces $X_{a}$ from $\mathcal{P}$, bimaps $p_{b}^{a}$ and $p^{a}$, and multi-valued [a]-bifunctions $J_{a}$. We shall define a multi-binet $\psi: Y \rightarrow X$ such that $\psi \circ f$ is bihomotopic to the identity multi-binet on $X$.

Let $a \in I n c^{b}(X)$. Since the function $f_{X_{a}}^{\#}$ is a surjection, there is a map $r^{a}: Y \rightarrow X_{a}$ with $p^{a} \simeq_{b} r^{a} \circ f$. Put $G_{a}=J_{a} \circ r^{a}$ and $\psi=\left\{G_{a}\right\}_{a \in \operatorname{Inc} b(X)}$. 
In order to verify that $\psi$ is a multi-binet, let a normal bicover $\sigma \in$ $\operatorname{Cov}^{b}(X)$ be given. Let $\tau \in \sigma^{*}$ and put $a=\{\tau\}$. For every $b \geq a$, we have

$$
r^{a} \circ f \simeq_{b} p^{a} \simeq_{b} p_{b}^{a} \circ p^{b} \simeq_{b} p_{b}^{a} \circ r^{b} \circ f .
$$

Since $f_{X_{a}}^{\#}$ is also an injection, we obtain $r^{a} \simeq_{b} p_{b}^{a} \circ r^{b}$. It follows that

$$
G_{a}=J_{a} \circ r^{a} \stackrel{\tau}{\simeq}_{b} J_{a} \circ p_{b}^{a} \circ r^{b} \stackrel{\tau}{\simeq}_{b} J_{b} \circ r^{b}=G_{b}
$$

and therefore $G_{a} \stackrel{\sigma}{\simeq}_{b} G_{b}$.

We shall now check that $\psi \circ f$.is bihomotopic to ${ }_{i} X$, i.e., that for every $\sigma \in \operatorname{Cov}^{b}(X)$ there is an $a \in \operatorname{Inc}^{b}(X)$ such that $G_{\psi(b)} \circ f \stackrel{\sigma}{\check{\sigma}_{b}} 1_{X}$ for every $b \geq a$.

Let $\sigma \in \operatorname{Cov}^{b}(X)$ and $\tau \in \sigma^{*}$. Put $a=\{\tau\}$. For every $b \geq a$, we get $\psi(b) \geq \psi(a) \geq a$ so that $G_{\psi(b)} \stackrel{\tau_{b}}{\sim_{b}} G_{\psi(a)}$. Hence,

$$
G_{\psi(b)} \circ f \stackrel{\tau}{\simeq}_{b} G_{\psi(a)} \circ f=J_{\psi(a)} \circ r^{\psi(a)} \circ f \stackrel{\tau}{\simeq}_{b} J_{\psi(a)} \circ p^{\psi(a)} \stackrel{\tau}{\simeq}_{b} 1_{X} .
$$

Thus, $G_{\psi(b)} \circ f \stackrel{\sigma}{\simeq_{b}} 1_{X}$.

Theorem 10. Let $\mathcal{P}$ and $\mathcal{R}$ be classes of bispaces such that each member of $\mathcal{R}$ is both internally movable and internally calm. If a bispace $X$ has a $\mathcal{P}$-exposition, a bispace $Y$ has an $\mathcal{R}$-exposition, and a bimap $f: X \rightarrow Y$ is such that the function $f_{Z}^{\#}$ is a bijection for every $Z \in \mathcal{P}$ and an injection for every $Z \in \mathcal{R}$, then $f$ is a bishape equivalence.

Proof. First we shall show that for every $a \in \operatorname{Cov}^{b}(Y)$ there is a $\tau \in \operatorname{Cov}^{b}(Y)$ such that the relation $U$ of $\underset{\sim}{\simeq} V \circ f$ for multi-valued $\tau$-bifunctions $U, V: Y \rightarrow Y$ implies the relation $U \stackrel{\sigma}{\simeq}_{b} V$.

Let a $\sigma \in \operatorname{Cov}^{b}(Y)$ be given. Let $\mu \in \sigma^{*}$ and $\xi \in \mu^{*}$. Put $c=\{\xi\}$. By assumption the bispace $Y$ has an $\mathcal{R}$-exposition formed by bispaces $Y_{d}$ from $\mathcal{R}$, bimaps $q_{e}^{d}$ and $q^{d}$, and multi-valued $[d]$-bifunctions $K_{d}$.

Since the bispace $Y_{c}$ is internally calm, there is a normal bicover $\alpha$ of $Y_{c}$ such that $\alpha$-bihomotopic bimaps into $Y_{c}$ are bihomotopic. Let $\beta \in \alpha^{*}$ be such that $K_{c}: Y_{c} \rightarrow Y$ is a multi-valued $(\beta, \xi)$-bifunction. 
Since $Y_{c}$ is also internally movable, there is a normal bicover $\gamma \in \beta^{+}$ of $Y_{c}$ with the property that every multi-valued $\gamma$-bifunction into $Y_{c}$ is $\beta$-bihomotopic to a bimap. Let $\tau=\left(q^{c}\right)^{-1}(\gamma)$.

Let $U, V: Y \rightarrow Y$ be multi-valued $\tau$-bifunctions and assume that $U \circ f \stackrel{\tau}{\simeq_{b}} V \circ f$. Then $q^{c} \circ U$ and $q^{c} \circ V$ are multi-valued $\gamma$-bifunctions so that there are bimaps $a$ and $b$ from $Y$ into $Y_{c}$ with $a \stackrel{\beta}{\simeq} q^{c} \circ U$ and $b \stackrel{\beta}{\simeq} q^{\mathrm{c}} \circ V$. Our choices imply the following chain of relations:

$$
a \circ f \stackrel{\beta}{\simeq}_{b} q^{c} \circ U \circ f \stackrel{\gamma}{\simeq}_{b} q^{c} \circ V \circ f \stackrel{\beta}{\simeq}_{b} b \circ f
$$

It follows that $a$ of $\simeq_{b} b \circ f$ and therefore $a \simeq_{b} b$ because $f_{Y_{c}}^{\#}$ is an injection. Now, we have

$$
U \stackrel{\mu}{\sim}_{b} K_{c} \circ q^{c} \circ U \stackrel{\mu}{\simeq}_{b} K_{c} \circ a \stackrel{\mu}{\sim_{b}} K_{c} \circ b \stackrel{\mu}{\simeq}_{b} K_{c} \circ q^{c} \circ V \stackrel{\mu}{\simeq}_{b} V .
$$

Hence, $U \stackrel{\sigma}{\simeq}_{b} V$.

Now we are ready to prove the theorem. Let $\psi$ be a multi-binet constructed in the proof of Theorem 9. We shall show that $f \circ \psi$ is bihomotopic to $i^{Y}$, i.e., that for every $\sigma \in \operatorname{Cov}^{b}(Y)$ there is an index $a \in I n c^{b}(Y)$ such that $f \circ G_{\psi_{\left(\varphi^{*}(b)\right)}} \stackrel{\sigma}{\sim}_{b} 1_{Y}$ for every $b \geq a$. Since we can take $\varphi^{*}(b)=\left\{f^{-1}([b])\right\}$, we must prove that $f \circ G_{\lambda(b)} \stackrel{g}{\sim}{ }_{b} 1_{Y}$ for every $b \geq a$, where $\lambda(b)=\psi\left(\left\{f^{-1}([b])\right\}\right)$.

Let a $\sigma \in \operatorname{Cov}^{b}(Y)$ be given. Choose a normal bicover $\tau$ of $Y$ with the property described above. Let $\varrho \in \tau^{*}$ and put $a=\{\varrho\}$. For every $b \geq a, f \circ G_{\lambda(b)}$ and $1_{Y}$ are multi-valued $\tau$-bifunctions of $Y$ into itself and

$$
f \circ G_{\lambda(b)} \circ f=f \circ J_{\lambda(b)} \circ r^{\lambda(b)} \circ f \stackrel{\varrho}{\simeq}_{b} f \circ J_{\lambda(b)} \circ p^{\lambda(b)} \stackrel{\ell}{\simeq}_{b} f=1_{Y} \circ f .
$$

It follows that $f \circ G_{\lambda(b)} \stackrel{a}{\sim}{ }_{b} 1_{Y}$. 


\section{APPROXIMATE CONTRACTIBILITY OF MAPS AND TRIVIAL BISHAPE}

As an illustration of the new insight offered in shape theory of maps by approach via multi-binets we will characterize bispaces of trivial bishape as approximately contractible bispaces.

Let us agree that a bispace $T$ is trivial provided both the codomain and the domain of $T$ are single-element spaces. A bispace $X$ has trivial bishape provided it is equivalent in the bishape category $\mathcal{H} M^{b}$ to a trivial bispace.

As we shall see shortly, bispaces of trivial bishape coincide with approximately contractible bispaces which we define next. A bispace $X$ is called approximately contractible provided for every normal bicover $\sigma$ of $X$ there is a normal bicover $\tau$ of $X$ such that every two multi-valued $\tau$-bifunctions into $X$ are $\sigma$-bihomotopic.

Theorem 11. A bispace $X$ is approximately contractible if and only if $X$ has trivial bishape.

Proof. ( $\Longrightarrow$ ). Let $T$ be a trivial bispace and let $c: X \rightarrow T$ be the obvious bimap. Let $a \in X$ and $a^{\prime} \in x^{-1}(a)$. Define a bimap $g: T \rightarrow X$ by $g(T)=a$ and $g^{\prime}\left(T^{\prime}\right)=a^{\prime}$. Let $\gamma: X \rightarrow T$ and $\psi: T \rightarrow X$ be simple multi-binets determined by bimaps $c$ and $g$, respectively. Then $\gamma \circ \psi=i^{T}$. In order to see that $X$ and $T$ are bishape equivalent, it remains to check that $\psi \circ \gamma \simeq_{b} i^{X}$, i.e., that $g \circ c \stackrel{\sigma}{\sim}{ }_{b} 1_{X}$ for every normal bicover $\sigma$ of $X$.

Let a $\sigma \in \operatorname{Cov}^{b}(X)$ be given. Since $X$ is approximately contractible, there is a $\tau \in \operatorname{Cov}^{b}(X)$ such that multi-valued $\tau$-bifunctions into $X$ are $\sigma$-bihomotopic. But, $g \circ c$ and $1_{X}$ are both multi-valued $\tau$-bifunctions of $X$ into itself. Hence, $g \circ c \stackrel{\sigma}{\sim} 1_{X}$.

$(\Longleftrightarrow$ ). It suffices to observe that every trivial bispace $T$ is approximately contractible and that approximate contractibility is preserved under bishape dominations and bishape equivalence.

Let us call a bimap $f: X \rightarrow Y$ constant provided both $f^{\prime}\left(X^{\prime}\right)$ and $f(X)$ are single points (i.e., maps $f^{\prime}$ and $f$ are constant). The following 
notion is a version of path-connectedness for maps. A bispace $X$ is pathconnected provided every two constant bimaps into $X$ are bihomotopic.

Theorem 12. If a bispace $X$ has trivial bishape, then every two bimaps from $X$ into a path-connected bispace which is both internally movable and internally calm are bihomotopic.

Proof. Let $Z$ be a path-connected bispace which is both internally movable and internally calm. Let $a, b: X \rightarrow Z$ be bimaps. Let $T$ be a trivial bispace. Since $X$ has trivial bishape, the obvious bimap $c: X \rightarrow T$ is a bishape equivalence. It follows from Theorem 7 that there are bimaps $f, g: T \rightarrow Z$ with $a \simeq_{b} f \circ c$ and $b \simeq_{b} g \circ c$. But, $f$ and $g$ are constant bimaps into $Z$ so that $f \simeq_{b} g$ because $Z$ is path-connected. Hence, $a \simeq_{b} b$.

Theorem 13. Let $\mathcal{P}$ be a class of path-connected bispaces. If a bispace $X$ has a $\mathcal{P}$-exposition and every two bimaps of $X$ into a member of $\mathcal{P}$ are bihomotopic, then $X$ has trivial bishape.

Proof. Let $c: X \rightarrow T$ be the obvious bimap from $X$ into a trivial bispace $T$. We shall show that $c$ is bishape left invertible. Then $X$ will be bishape dominated by the approximately contractible bispace $T$ and will therefore have trivial bishape by Theorem 11. According to Theorem 9, we must in fact check that the induced function $c_{Z}^{\#}$ is a bijection for every $Z \in \mathcal{P}$.

In order to see that $c_{Z}^{\#}$ is a surjection for every $Z \in \mathcal{P}$, let $Z$ be from $\mathcal{P}$ and let $f: X \rightarrow Z$ be a bimap. Let $g: T \rightarrow Z$ be a constant bimap. Then $f$ and $g \circ c$ are two bimaps of $X$ into $Z$. By assumption, they are bihomotopic so that $[f]_{b}=c_{Z}^{\#}\left([g]_{b}\right)$ and $c_{b}^{\#}$ is onto.

Finally, in order to see that $c_{Z}^{\#}$ is an injection for every $Z \in \mathcal{P}$, let $Z$ be from $\mathcal{P}$ and let $f, g: T \rightarrow Z$ be bimaps and assume that $c_{Z}^{\#}\left([f]_{b}\right)=c_{Z}^{\#}\left([g]_{b}\right)$, i.e., that $f \circ c \simeq_{b} g \circ c$. But, the bimaps $f$ and $g$ are constant bimaps into $Z$. Since $Z$ is path-connected, we obtain $f \simeq_{b} g$ so that $c_{Z}^{\#}$ is indeed one-to-one. 


\section{References}

[1] Aló, R.A. and Shapiro, M.L., Normal Topological Spaces, Cambridge Univ. Press, Cambridge, 1972.

[2] Baladze, V.H., Fiber shape theory of maps and resolutions, Bull. Acad. Sci. of Georgian SSR 141 (1991), 489-492.

[3] Borsuk, K., Concerning homotopy properties of compacta, Fund. Math. 62 (1968), 223-254.

[4] Cerin, Z., Shape theory intrinsically, Publicacions Mathemàtiques 37 (1993), 317-334.

[5] Dold, A., Lectures on Algebraic Topology, Springer-Verlag, Berlin, 1972.

[6] Edwards, D.A. and McAuley, P.T., The shape of a map, Fund. Math. 96 (1977), 195-210.

[7] Kozlowski, G., Images of ANR's, Trans. Amer. Math. Soc. (to appear).

[8] Mardešić,S. and Segal, J., Shape Theory, North Holland, Amsterdam, 1982.

[9] Sanjurjo, J., An intrinsic description of shape, Trans. AMS 329 (1992), 625-636.

[10] Spanier, E.H., Algebraic Topology, McGraw-Hill, New York, 1966.

Kopernikova 7, 41020 Zagreb,

Recibido: 30 de Noviembre de 1993

Crontia, Europe

E-mail address: zcerin 1400 .SRCE.HR 